\title{
Bewegungsförderung in Deutschland: Akteure, Strukturen und Netzwerkentwicklung
}

\section{Physical activity promotion in Germany: Actors, structures and network development}

\author{
Autoren \\ Hagen Wäsche ${ }^{1}$, Stefan Peters ${ }^{2}$, Luisa Appelles ${ }^{1}$, Alexander Woll ${ }^{1}$ \\ Institute \\ 1 Institut für Sport und Sportwissenschaft, Karlsruher Institut \\ für Technologie (KIT) \\ 2 Deutscher Verband für Gesundheitssport und Sporttherapie \\ e. V. (DVGS)
}

Stichworte

Bewegungsförderung, Strukturen, Stakeholder, Multiplikatoren, Netzwerkanalyse

Key words

Physical activity promotion, structure, stakeholder, multiplicator, network analysis

Eingegangen: 12.03.2018

Angenommen durch Review: 23.08.2018

Bibliografie

DOI https://doi.org/10.1055/a-0739-9857

Bewegungstherapie und Gesundheitssport 2018; 34: 257-273

(C) Georg Thieme Verlag KG

ISSN 1613-0863

\section{ZUSAMMENFASSUNG}

Bewegung unterstützt in vielfältiger Weise die Gesundheit von Menschen jeden Lebensalters. Dennoch ist die Prävalenz körperlicher Inaktivität in Deutschland hoch und die meisten Menschen erreichen den von der Weltgesundheitsorganisation empfohlenen Bewegungsumfang nicht. Um gezielte Maßnahmen zur Bewegungsförderung zu unterstützen, ist die Kenntnis der relevanten Akteure, Berufsgruppen und Multiplikatoren sowie deren struktureller Zusammenhang notwendig. Im Rahmen einer explorativen Untersuchung wurden 2 Ziele verfolgt: (1) die Identifikation relevanter Akteure, Berufsgruppen und Multiplikatoren der Bewegungsförderung in Deutschland und (2) die Analyse der strukturellen Vernetzung dieser Akteure sowie die Formulierung von Empfehlungen zur nachhaltigen Entwicklung eines nationalen Netzwerks der Bewegungsförderung. Hierzu wurden qualitative Experteninterviews, eine anschließende Netzwerkvisualisierung und eine Analyse zur Netzwerkentwicklung durchgeführt. Es zeigte sich, dass das Feld der
Akteure im Bereich der Bewegungsförderung ausgesprochen heterogen und umfangreich hinsichtlich verschiedener Akteursformen, Gesellschaftsbereiche und Bezugsräume ist. Insgesamt konnten 128 Akteure identifiziert werden. Davon werden 22 Akteure in Schlüsselpositionen gesehen. Bei den Multiplikatoren wurden 19 aktuelle und 17 weitere potenzielle identifiziert. Die strukturelle Vernetzung der Akteure ist nur schwach ausgeprägt. Für eine zukünftige Netzwerkentwicklung konnten verschiedene Voraussetzungen, Vorteile und Barrieren herausgearbeitet werden. Daran anschließend werden Empfehlungen formuliert, welche zur Bildung und effektiven Steuerung eines nationalen Netzwerks der Bewegungsförderung beitragen. Die Studie ermöglicht eine erste differenzierte Betrachtung der Akteursstrukturen der Bewegungsförderung in Deutschland.

\section{SUMMARY}

Physical activity supports the health of human beings of every age group in many ways. However, the prevalence of physical inactivity in Germany is high and most people do not reach the amount of physical activity that is recommended by the World Health Organization. To support targeted measures of physical activity promotion, it is necessary to gain knowledge of relevant actors, professionals groups and multiplicators as well as their structural connection. In an explorative study, 2 goals were addressed: (1) the identification of relevant actors, professional groups and multiplicators of physical activity promotion in Germany, and (2) the analysis of structural relations among these actors as well as the formulation of recommendations for the sustainable development of a national network of physical activity promotion. For that purpose, qualitative expert interviews, a subsequent network visualization and an analysis for network development were carried out. It became apparent that the field of actors in the area of physical activity promotion is very heterogeneous and extensive with regard to different forms of actors, sectors of society and administrative levels. Overall, 128 actors were identified, of which 22 actors are considered to hold key positions. Concerning the multiplicators, 19 current and 17 potential ones were identified. Structural relations among actors are sparse. For future network development, 
various prerequisites, benefits, and barriers were revealed. Subsequently, recommendations that contribute to the development and effective governance of a national network of physical activity promotion are given. The study provides a first detailed consideration of the structure of physical activity promotion in Germany.
WAS IST ZU DIESEM THEMA BEREITS BEKANNT?

- Bewegungsförderung bedarf gezielter Maßnahmen

- die Einbindung und Vernetzung der Akteure der Bewegungsförderung stellt die Grundlage einer koordinierten und sektorenübergreifenden Bewegungsförderungsstrategie dar

- die systematische Identifikation relevanter Akteure, Berufsgruppen und Multiplikatoren der Bewegungsförderung in Deutschland ist für eine gezielte Maßnahmenentwicklung notwendig

\section{WELCHE NEUEN ERKENNTNISSE BRINGT DER}

ARTIKEL?

- die Landschaft der relevanten Akteure ist ausgesprochen heterogen und umfassend

- neben relevanten Akteuren wurden Schlüsselakteure, Multiplikatoren und potenzielle Multiplikatoren identifiziert

- die strukturelle Vernetzung der Akteure ist schwach

- auf Grundlage einer Netzwerkanalyse werden Handlungsempfehlungen zur Bildung und Steuerung eines Netzwerks der Bewegungsförderung formuliert

\section{Einleitung}

\section{HINWEIS}

In diesem Beitrag werden die zentralen Begriffe des Akteurs und Multiplikators im generischen Maskulinum verwendet, um männliche und weibliche Personen genauso wie geschlechtsneutrale Organisationen und Einrichtungen zu bezeichnen. Zur besseren Lesbarkeit wurde zudem bei bestimmten Personengruppen die männliche Form gewählt, welche alle Geschlechter umfasst.

In zahlreichen Studien wurde nachgewiesen, dass Bewegung in vielfältiger Weise die Gesundheit von Menschen jeden Lebensalters unterstützt [12,14]. Dennoch ist die Prävalenz körperlicher Inaktivität in Deutschland hoch und die meisten Menschen erreichen den von der Weltgesundheitsorganisation empfohlenen Bewegungsumfang [20] nicht [8]. Um vorhandene Barrieren abzubauen und individuelle sowie strukturelle Ressourcen für Bewegung zu stärken, sind gezielte Maßnahmen zur Bewegungsförderung notwendig.

Im Anschluss an das Präventionsgesetz wurden von der Nationalen Präventionskonferenz im Februar 2016 Bundesrahmenempfehlungen mit dem Ziel, die Qualität der Gesundheitsförderung und Prävention zu sichern und weiterzuentwickeln, verabschiedet. Im
Fokus steht die „(...) Zusammenarbeit der für die Erbringung von Leistungen zur Gesundheitsförderung und Prävention in Lebenswelten und in Betrieben zuständigen Träger und Stellen insbesondere durch Festlegung gemeinsamer Ziele, vorrangiger Handlungsfelder und Zielgruppen, zu beteiligender Organisationen und Einrichtungen (...)“ [5]. Im September 2016 wurden die Nationalen Empfehlungen für Bewegung und Bewegungsförderung (NEBB) veröffentlicht, welche sich an professionelle Akteure und Organisationen der Bewegungsförderung richtet, um diese in ihrer Arbeit zu unterstützen [15]. Dies stellt einen wichtigen Schritt in Richtung einer systematischen und flächendeckend effektiven Bewegungsförderung in Deutschland dar.

Eine Disseminierung dieser Empfehlungen setzt jedoch die Kenntnis der Stakeholder bzw. der relevanten Akteure voraus, welche in Deutschland einen direkten oder indirekten Beitrag zur Bewegungsförderung leisten. Des Weiteren stellt die Einbindung und Vernetzung dieser Akteure die Grundlage einer koordinierten, sektorenübergreifenden Zusammenarbeit in der Bewegungsförderung als gesamtgesellschaftliche Aufgabe dar [16]. Die Weltgesundheitsorganisation verweist explizit auf die Bedeutung eines die Akteure vernetzenden und koordinierten Ansatzes einer nationalen Bewegungsförderungsstrategie [21]. Allerdings ist die Gruppe dieser Akteure sowie ihrer Verbindungen ausgesprochen vielfältig und heterogen. Um einen systematischen Überblick über diese Akteurslandschaft zu gewinnen, bedarf es einer Analyse der vorhandenen Akteursstrukturen (vgl. auch [7]). Hierzu wurde vom Bundesgesundheitsministerium im Juli 2016 das Projekt SAMBA (Systematische Erfassung relevanter Akteure, Berufsgruppen sowie künftiger Multiplikatoren in der Bewegungsförderung zur Analyse und Entwicklung eines interdisziplinären Netzwerks zur nachhaltigen Bewegungsförderung) initiiert.

Das übergeordnete Ziel dieses explorativen Projekts war es, einen umfassenden Überblick über Akteure, Berufsgruppen und Multiplikatoren der Bewegungsförderung zu geben sowie Empfehlungen zur nachhaltigen Entwicklung eines Netzwerks der Bewegungsförderung zu formulieren. Die Abbildung und das Aufzeigen des Entwicklungspotenzials dieser „Landschaft der Bewegungsförderung“ soll des Weiteren einen Beitrag zu einer flächendeckenden und nachhaltigen Disseminierung der NEBB leisten. Einem sozialökologischen Ansatz folgend, werden dabei alle das individuelle Bewegungsverhalten beeinflussenden Umweltfaktoren (interpersonal, organisational, kommunal, politisch) berücksichtigt [17].

Das Projekt SAMBA umfasste 2 Teilprojekte [11]. Einerseits wurden Experteninterviews und eine anschließende Netzwerkanalyse durchgeführt. Andererseits erfolgte eine Fragebogenbefragung verschiedener Akteure der Bewegungsförderung in Deutschland, wobei diese Befragung einen additiven Charakter besitzt (Peters et al., in diesem Heft). Die auf den Experteninterviews basierende Untersuchung ist der Gegenstand dieses Beitrags und umfasste 2 Ziele. Das 1. Ziel bestand in der explorativen Erfassung der Akteure, 
Berufsgruppen und Multiplikatoren für die Entwicklung eines flächendeckenden Netzwerks der Bewegungsförderung. Die zugrundeliegende Fragestellung lautete, wer die relevanten Akteure, die Akteure in Schlüsselpositionen und aktuelle sowie potenzielle Multiplikatoren der Bewegungsförderung in Deutschland sind. Das 2. Ziel war eine Analyse der strukturellen Vernetzung der Akteure sowie die Erarbeitung von Empfehlungen zur nachhaltigen Netzwerkentwicklung. Die Frage hierbei war, wie die identifizierten Akteure der Bewegungsförderung in Deutschland miteinander vernetzt sind und welche Bedingungen für eine Netzwerkentwicklung förderlich sind.

\section{Methode}

Zur Untersuchung wurden sowohl etablierte Methoden der empirischen Sozialforschung, als auch Methoden der sozialen Netzwerkanalyse [19] in einem neuartigen Mixed-Methods-Ansatz kombiniert.

Im 1. Schritt kamen leitfadengestützte Experteninterviews [6] zum Einsatz, um Wissensbestände und Einschätzungen zu relevanten Akteuren, Berufsgruppen sowie Multiplikatoren zu erschließen. Der Interviewleitfaden umfasste folgende Fragenkomplexe:

- Wer sind die wichtigsten Akteure der Bewegungsförderung von nationaler und überregionaler Relevanz in Deutschland?

- Wie ist deren Verständnis von Bewegung und Bewegungsförderung? Wie sind deren Funktion und Kompetenzen hinsichtlich der Bewegungsförderung? Wer ist deren Zielgruppe?

- Welcher dieser Akteure nimmt dabei eine übergeordnete Schlüsselposition ein und wodurch zeichnet sich diese aus?

- Welche der Akteure sind wichtigsten Multiplikatoren oder haben das Potenzial dafür?

- Stehen diese Akteure in einem Beziehungszusammenhang?

- Welche Vor- und Nachteile bietet eine Beteiligung an einem nationalen Netzwerk der Bewegungsförderung?

Auf Grundlage der Expertensicht soll die nationale Landschaft der Bewegungsförderung nachgezeichnet werden. Neben der Identifizierung relevanter Akteure und deren Beitrag zur Bewegungsförderung können so deren Position und Rolle in der Bewegungsfördererlandschaft sowie Form und Inhalte bestehender Vernetzungen zwischen den relevanten Akteuren, Berufsgruppen und Multiplikatoren identifiziert werden. Die Auswertung der Interviews erfolgte durch eine strukturierende, qualitative Inhaltsanalyse mittels Entwicklung eines Kategoriensystems [9].

Im 2. Schritt wurde mithilfe einer Netzwerkanalyse, welche qualitative und quantitative Methoden kombiniert [4], das Netz relevanter Akteure der Bewegungsförderung dargestellt. Dies ermöglicht eine weitergehende Netzwerkanalyse und die Erstellung einer Netzwerkkarte zur strukturellen Einbettung individueller Akteure und übergeordneter struktureller Zusammenhänge [1]. Daraus lassen sich Schlüsse ziehen, welche die Basis einer Netzwerkentwicklung im Sinne einer Beseitigung struktureller Defizite und einer nachhaltigen Entwicklung bilden. Unter Berücksichtigung der spezifischen Netzwerkkonfigurationen können so Empfehlungen zur Netzwerkgovernance [13] entwickelt werden, welche eine effektive Netzwerksteuerung und nachhaltige Netzwerkentwicklung zur Disseminierung der NEBB unterstützen sollen.
Bei der Auswahl der Experten wurde darauf geachtet, dass ein möglichst weites Spektrum der Gesellschaftsbereiche der Bewegungsförderung abgebildet wird. In gemeinsamer Abstimmung des Projektteams wurden folgende Gesellschaftsbereiche als relevant erachtet und als Grundlage der Expertenauswahl genutzt:

- Bildung \& Forschung: Universität, Hochschule, Fachschule, Schule, Kindergarten etc.

- Gesundheit: Ärztevertretungen und Leistungsträger, Prävention und Gesundheitsförderung, Rehabilitation, Pflege, Gesundheitssport etc.

- Politik \& Verwaltung: Akteure auf Bundes- und Landesebene, Kommunalverwaltung etc.

- Sport: Organisierter Sport, Leistungs- und Breitensport, Sportwissenschaft etc.

- Wirtschaft: Sportartikel / -geräte, Landschafts- und Stadtplanung, Architektur etc.

Die Fallauswahl fand im Rahmen eines theoretischen Samplings anhand begründeter Vorüberlegungen statt. Die Größe des Samples wurde aufgrund des Anspruchs einer inhaltlichen Sättigung bewusst offen gestaltet, indem nicht von vorneherein festgelegt wurde, wie viele Experten interviewt werden sollten [3]. Erst als davon auszugehen war, dass keine weiteren gewinnbringenden Erkenntnisse erlangt werden können, wurde das Sample geschlossen.

Die Auswahl der Interviewpartner deckte zunächst Experten aus allen 5 als relevant definierten Gesellschaftsbereichen ab. Zudem wurde innerhalb des Projektteams vor der Auswahl und Kontaktaufnahme umfassend der Expertenstatus der ausgewählten Personen diskutiert, um sicherzustellen, dass Informationen hoher Qualität generiert werden können. Nach der Durchführung und Analyse der 1. Interviews wurde deutlich, dass zusätzliche Interviews einen weiteren Erkenntnisgewinn erwarten lassen. In einem iterativen Prozess wurden daraufhin weitere Interviewpartner ausgewählt und befragt, bis ein zufriedenstellender inhaltlicher Sättigungsgrad erreicht wurde.

Insgesamt wurden 10 Experten interviewt, wobei jeweils 2 einem der oben genannten Gesellschaftsbereiche entstammten. Experten in Doppel- oder Mehrfachfunktionen wurden gemäß ihrer hauptberuflichen Funktion zugeordnet. Die Zusammensetzung der Experten in Bezug auf die Gesellschaftsbereiche gliederte sich wie folgt:

- Bildung \& Forschung: Schule, Universität $(n=2)$

- Gesundheit: Gesundheitssport, Prävention / Gesundheitsförderung $(n=2)$

- Politik \& Verwaltung: Bundes- / Landesebene, Kommunalebene $(n=2)$

- Sport: Organisierter Sport, Sportwissenschaft $(n=2)$

- Wirtschaft: Landschafts- und Stadtplanung, Sportartikel und -geräte $(n=2)$

4 der Befragten waren weiblich und 6 waren männlich. Alle Experten besetzen leitende Funktionen (teilweise mehrere in Personalunion) in den durch sie vertretenen Einrichtungen.

Die Experteninterviews wurden im Zeitraum November 2016 bis Februar 2017 geführt. Im Durchschnitt dauerte ein Interview knapp 54 Minuten (Range: 31-72 Minuten). Die Interviews wurden im Anschluss wortgetreu transkribiert. Die Befragten werden in den transkribierten Interviews mit den Kürzeln „B1“ bis „B10“ bezeichnet, der Interviewer mit dem Kürzel „““. 
Durch ein mehrfaches Bearbeiten des vorliegenden Materials in einem zirkulären Prozess wurden letztendlich 297 Kategorien und Subkategorien entwickelt. Die Festlegung der Hauptkategorien orientierte sich dabei an folgenden Dimensionen:

- Relevante Akteure

- Schlüsselpositionen

- Multiplikatoren

- Potenzielle Multiplikatoren

- Beziehungszusammenhang der Akteure (Kooperation, Konflikt / Konkurrenz)

- Netzwerkentwicklung (Nutzen und Barrieren der Netzwerkbeteiligung)

Während die Hauptkategorien deduktiv anhand der Fragestellungen abgeleitet wurden, entwickelten sich die Subkategorien induktiv im Verlauf der Inhaltsanalyse [9]. Zur Anwendung kamen hierbei die Softwarepakete MaxQDA und f4analyse.

\section{Ergebnisse}

\section{Akteure der Bewegungsförderung}

Bei der Identifizierung relevanter Akteure wiesen viele der Experten unterschiedliche Verständnisse auf und nahmen dementsprechend unterschiedliche Spezifizierungen der Akteure vor. Um diesem Umstand gerecht zu werden, werden im Folgenden verschiedene Akteursformen unterschieden:

- Individueller Akteur: einzelne Personen, Organisationen, Einrichtungen etc.

- Kollektiver Akteur: Gruppe individueller Akteure, die gemeinsame Ziele und Interessen verfolgen, häufig formal organisiert
- Akteursaggregat: inhomogene Gruppe individueller Akteure mit partiell gemeinsamen Zielen

- Berufsgruppe: Gruppe individueller Akteure, die formell oder informell als Vertreter eines Berufstands auftreten

- Soziale Institutionen: Regelsystem zum Lösen gesellschaftlicher Probleme, i. e. S. gesellschaftliche Einrichtungen wie die Schule, der Verein oder die Kommune

Zudem lassen sich die Akteure unterschiedlichen Bezugsräumen zuordnen. Dies betrifft für den Untersuchungsraum Deutschland die nationale Ebene, die Landesebene und zusammengefasst die Regional- und Kommunalebene.

\section{Relevante Akteure}

Aus den Interviews gingen insgesamt 128 relevante Akteure der Bewegungsförderung in Deutschland hervor. Diese lassen sich folgenden Gesellschaftsbereichen zuordnen:

- Bildung \& Forschung

- Familie, Soziales \& Umwelt

- Gesundheit

- Medien

- Politik \& Verwaltung

- Wirtschaft

- Sport

Die Bereiche Familie, Soziales und Umwelt wurden aufgrund der geringen Anzahl vorliegender Akteursnennungen und zur übersichtlicheren Darstellung zusammengefasst. Die Zuordnung zu Gesellschaftsbereichen sowie den zuvor genannten Bezugsräumen erlaubt eine strukturierte Betrachtung der identifizierten Akteure.

In > Abb. $\mathbf{1}$ ist die Verteilung der Häufigkeit genannter Akteure zunächst nach Gesellschaftsbereichen dargestellt (mehrfache

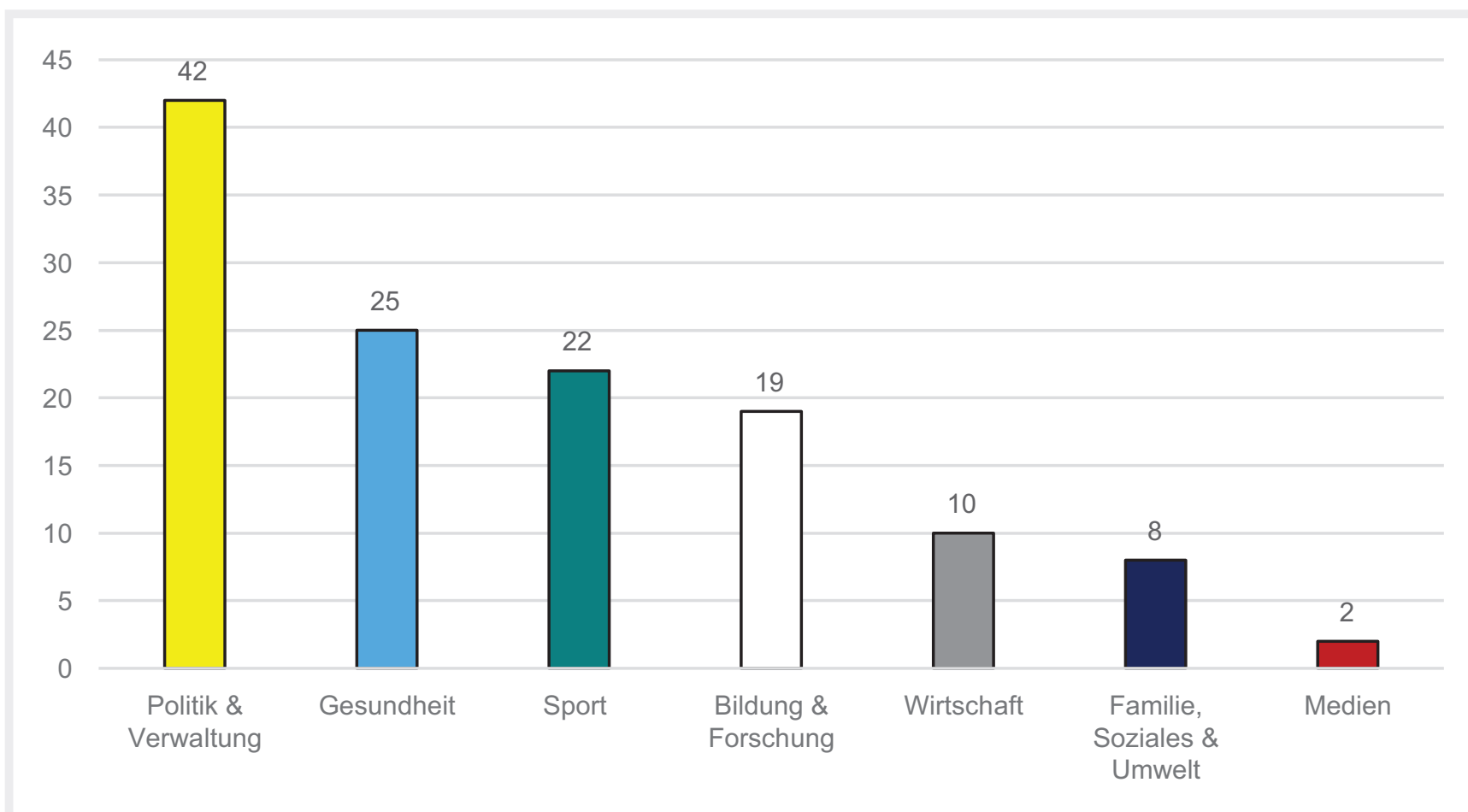

-Abb. 1 Verteilung relevanter Akteure nach Gesellschaftsbereichen (nach Anzahl der Nennungen), N=128. 
Nennungen über alle Interviews hinweg wurden lediglich einmal berücksichtigt).

Auffällig ist die hohe Anzahl genannter Akteure aus dem Bereich Politik und Verwaltung mit 42 verschiedenen Akteuren wie Kommunen und deren Einrichtungen oder verschiedene Ministerien auf Bundes- und Landesebene. Darauf folgt nach Anzahl der genannten Akteure der Gesellschaftsbereich der Gesundheit $(n=25)$. Diesem Bereich wurden u. a. Krankenkassen, Präventionsverbände und Berufsgruppen im Bereich des Gesundheitswesens zugeordnet. Der Bereich des Sports folgt an 3. Stelle $(n=22)$, u. a. vertreten durch den Deutschen Olympischen Sportbund (DOSB), Landessportverbände und einzelne Sportfachverbände (z.B. Deutscher Turnerbund (DTB), Deutscher Skiverband (DSV), Deutscher Tanzsportverband (DTV)). Akteure aus dem Bereich der Bildung und Forschung (z. B. Universitäten, Schulen, Kindergärten) wurden 19-mal genannt und befinden sich damit anteilsmäßig im mittleren Bereich der relevanten Akteure der Bewegungsförderung. Weniger häufig wurden Akteure aus den Bereichen Wirtschaft (z. B. Lebensmittelhersteller, Sportartikelindustrie), Familie, Soziales \& Umwelt (z. B. Kirche, Familie, Naturschutzbund) und Medien (Medienvertreter, Schauspieler) genannt.

Eine Gewichtung der Akteure lässt sich vornehmen, indem man die 14 Akteure herausgreift, die von mindestens der Hälfte der befragten Experten als relevante Akteure der Bewegungsförderung in Deutschland angeführt wurden (vgl. > Tab. 1.1). Hierbei ist zu berücksichtigen, dass es sich um die Häufigkeit der Nennung im Rahmen einer qualitativen Analyse handelt. Die Zahlen sind nicht repräsentativ, sondern geben die Perspektive der befragten Experten wieder. Eine herausragende Position nimmt demnach der Deutsche Olympische Sportbund (DOSB) ein, welcher von 9 der 10 Experten und damit am häufigsten genannt wurde. Daneben fällt auf, dass es sich bei vielen der Akteure um soziale Institutionen wie die Universität, die Schule, den Kindergarten, den Sportverein und die Kommune handelt.

Die in der Gesamtsicht der Experten 14 zentralen Akteure entstammen zum einem dem Bereich der Politik und Verwaltung wie das Bundesministerium für Gesundheit (BMG), Akteure / Ministerien auf Landesebene, der Deutsche Städtetag und die Kommune. Bildung und Forschung sind durch Kindergarten, Schule und Universität/Hochschule vertreten. Der Bereich des Sports ließ sich für eine differenzierte Betrachtung auf der einen Seite in einen Non-Profit-Bereich mit den Akteuren Deutscher Olympischer Sportbund (DOSB), Landessportbund/ Landessportverband (LSB / LSV) sowie dem Sportverein und auf der anderen Seite in einen For-Profit-Bereich mit der Fitnessbranche, Fitnessstudios und kommerziellen Sportanbietern unterteilen. Die Krankenkassen sind gemäß dieser Gewichtung die einzigen Vertreter des Bereichs Gesundheit. Akteure aus den Bereichen Wirtschaft, Medien oder Familie, Soziales \& Umwelt finden sich nicht unter den am häufigsten genannten relevanten Akteuren.

\section{Schlüsselpositionen}

Auf die Frage nach Akteuren, die zentrale Schlüsselpositionen in der Bewegungsförderung besetzen, wurden insgesamt 22 Akteure angeführt (vgl. \ Tab. 1.2). Dabei wurde der Begriff der Schlüsselposition zunächst nicht spezifiziert, sondern den Experten die Entscheidung darüber übertragen, welcher Akteur eine Schlüsselposition besetzt. Hier wurden häufig spezifische, individuelle Akteure, wie der Bundesgesundheitsminister oder die Bundeszentrale für gesundheitliche Aufklärung (BZgA) genannt. Anders als bei kollektiven Akteuren wie dem Arbeitgeber / Betrieb oder sozialen Institutionen wie Schulen handelt es sich um klar einzugrenzende und ansprechbare Akteure, die in Form einer Person oder Organisation auftreten.

Hierbei finden sich neben dem zuvor schon aufgeführten DOSB andere Verbände aus den Bereichen Sport und Gesundheit. Zudem wurden viele Akteure der bundespolitischen Ebene wie das BMI, die BZgA und das ebenfalls zuvor schon aufgeführte BMG angeführt. Ein Experte beschrieb die Schlüsselposition des BMG hierbei insbesondere als Initiator einer politischen Agenda:

„Im Moment ist es das Bundesgesundheitsministerium, die ja letzten Endes gerade all diese Aktivitäten [anstoßen]. Wie die, welche jetzt unter anderem auch zu diesem Gespräch und zu den Bewegungsempfehlungen geführt haben, und damit eine Policy für Bewegungsförderung einfach initiieren." $(B 7,83)$

Bei den Schlüsselpositionen wurden einzelne herausragende Personen aus dem Bereich der Politik und Verwaltung wie die Bundeskanzlerin oder 2 Akteure des BMG, der zum Untersuchungszeitpunkt amtierende Bundesgesundheitsminister Hermann Gröhe und Ute Winkler (BMG, Ref. 422), genannt, die aufgrund der

$\checkmark$ Tab. 1.1 Relevante Akteure der Bewegungsförderung (Nennungen $\geq 5, n=14$ ).

\begin{tabular}{|l|l|l|l|l|}
\hline Bezugsraum & $\begin{array}{l}\text { Politik \& } \\
\text { Verwaltung }\end{array}$ & Sport (Non-Profit) & Sport (For-Profit) & Gesundheit \\
\hline Nationale Ebene & $\begin{array}{l}\text { BMG, Deutscher } \\
\text { Städtetag }\end{array}$ & DOSB & Krankenkasse \\
\hline Landesebene & $\begin{array}{l}\text { Akteure / Ministe- } \\
\text { rien auf } \\
\text { Landesebene }\end{array}$ & LSB / LSV & \\
\hline $\begin{array}{l}\text { Regionale / Kom- } \\
\text { munale Ebene }\end{array}$ & $\begin{array}{l}\text { Kommerane } \\
\text { Sportverein }\end{array}$ & $\begin{array}{l}\text { Fitnessstudio, } \\
\text { Kommerzielle } \\
\text { Sportanbieter }\end{array}$ \\
\hline
\end{tabular}


-Tab. 1.2 Schlüsselpositionen der Bewegungsförderung ( $n=22)$.

\begin{tabular}{|c|c|c|c|c|c|c|}
\hline Bezugsraum & $\begin{array}{l}\text { Politik \& } \\
\text { Verwaltung }\end{array}$ & $\begin{array}{l}\text { Sport (Non- } \\
\text { Profit) }\end{array}$ & Gesundheit & $\begin{array}{l}\text { Bildung \& } \\
\text { Forschung }\end{array}$ & $\begin{array}{l}\text { Wirt- } \\
\text { schaft }\end{array}$ & $\begin{array}{l}\text { Familie, Soziales } \\
\text { \& Umwelt }\end{array}$ \\
\hline Nationale Ebene & $\begin{array}{l}\text { Bundesgesund- } \\
\text { heitsminister H. } \\
\text { Gröhe, BMG, Bun- } \\
\text { deskanzlerln, BMI, } \\
\text { BZgA, U. Winkler } \\
\text { (BMG) }\end{array}$ & DBS, DOSB, DTB & $\begin{array}{l}\text { DVGS, } \\
\text { Krankenkassen }\end{array}$ & $\begin{array}{l}\text { BISp, Wissen- } \\
\text { schaftsakteure / } \\
\text { Wissenschaft }\end{array}$ & & \\
\hline Landesebene & & & $\begin{array}{l}\text { Landesvereini- } \\
\text { gungen } \\
\text { Gesundheit }\end{array}$ & & & \\
\hline $\begin{array}{l}\text { Regionale / Kom- } \\
\text { munale Ebene }\end{array}$ & Kommune, SVLP & Sportverein & Ärzte & Schule, VHS & $\begin{array}{l}\text { Arbeit- } \\
\text { geber / } \\
\text { Betrieb }\end{array}$ & Familie \\
\hline
\end{tabular}

ihnen zugeschriebenen politischen Macht und Einflussmöglichkeiten als Schlüsselakteure der Bewegungsförderung in Deutschland betrachtet werden. Dabei wurde die Schlüsselrolle des Bundesgesundheitsministers in der Bewegungsförderung auch kritisch gesehen und auf hier bestehendes Entwicklungspotenzial hingewiesen: „(...) also aus meiner persönlichen Sicht ist jemand in diesem Spiel drin, der eine richtig große Rolle übernehmen könnte, der das aber nicht tut. Das ist der Bundesgesundheitsminister" (B5, 81). Ähnlich wurde auch die Rolle der Bundeskanzlerin betrachtet, welche aufgrund ihrer Position dem Thema Bewegungsförderung in Deutschland zu einem höheren Stellenwert verhelfen könnte.

Neben Akteuren und Institutionen auf Bundesebene wurden viele lebensweltliche Settings der Bewegungsförderung auf kommunaler Ebene wie der Betrieb, die Schule, die VHS und der Sportverein als Schlüsselpositionen bezeichnet. In diesem Zusammenhang wurde die große Bedeutung der Familie als entscheidender Einflussfaktor für Bewegung in früher Kindheit betont. Eine übergeordnete Schlüsselposition besitzt die Kommune, welche alle lebensweltlichen Settings der Bürger umfasst und die Möglichkeit gibt, bundes- oder landespolitische Maßnahmen bürgernah umzusetzen.

Neben der Kommune, welche eine koordinative, sektorenübergreifende Funktion hat, wurden die Akteure der Stadt-, Verkehrsund Landschaftsplanung genannt, die den Bewegungsraum der Kommune maßgeblich gestalten und zur Bewegungsförderung beitragen. Als einzige explizite Berufsgruppe wurden die Ärzte angeführt, welche aufgrund ihres direkten Kontakts mit einem großen Teil der Bevölkerung über eine Schlüsselposition mit hohen Einflussmöglichkeiten verfügen. Weiterhin wird den Landesvereinigungen für Gesundheit, dem BISp und den Wissenschaftsakteuren als Produzenten des für die Bewegungsförderung relevanten Wissens eine Schlüsselposition zugewiesen.

Betrachtet man die Schlüsselpositionen im Hinblick auf die Verteilung nach Gesellschaftsbereichen, fällt auf, dass das Gros der Akteure dem Bereich der Politik und Verwaltung, v. a. der nationalen Ebene, entstammt.

\section{Multiplikatoren}

Im Kontext der Untersuchung werden Multiplikatoren als Akteure, die Wissen oder Informationen zu Bewegungsförderung weitergeben und dadurch zur Verbreitung dieses Wissens beitragen, verstanden. In den Interviews wurden insgesamt 19 Multiplikatoren genannt (vgl. $>$ Tab. 1.3).

Neben unspezifischen Akteursaggregaten wie Politiker/Vertreter der Politik in der Öffentlichkeit oder die Akteure/Ministerien auf Landesebene wurde eine große Zahl spezifischer, individueller Akteure genannt. Abermals handelt es sich dabei v. a. um politische bzw. verwaltende Organisationen und Sportverbände auf nationaler Ebene, wie z. B. den DAV, den DWV und die dvs.

Zudem fällt auf, dass viele der Multiplikatoren wie der DOSB, das BMG oder die BZgA bereits zuvor als Akteure in Schlüsselpositionen genannt wurden. Die bereits bestehende Multiplikatorenrolle der $\mathrm{BZgA}$ wurde von einer Expertin folgendermaßen beschrieben:

"Ja und das Gleiche soll jetzt im Rahmen dieses Präventionsgesetzes auch die BZgA machen, also als Beratung der Kommunen und als Multiplikator. Diesen Auftrag hatten sie ja als Institut vorher auch schon. Und da muss man jetzt auch eine gute Zusammenarbeit [etablieren] und in ein gutes System kommen, wie man sich da auch wirklich gegenseitig darüber informiert, was eigentlich gebraucht wird." (B9, 93)

In diesem Zusammenhang verweist sie auf die Bedeutung der inhaltlichen Vernetzung und intensiven Kooperation als Voraussetzung für die Funktion des Multiplikators.

Darüber hinaus wurden etliche Akteure genannt, die als Multiplikator eine Brückenfunktion zwischen den Ebenen einnehmen. So kommt etwa den Akteuren / Ministerien auf Landesebene eine Vermittlerrolle zwischen Bund und Kommune zu. Im Bereich des Sports nehmen LSB eine ähnliche Rolle ein. Ein anderes Beispiel ist der Deutsche Städtetag, über den der Weg zu den Kommunen führt. Weitere Multiplikatoren sind kommunale Verwaltungsbehörden 
-Tab. 1.3 Multiplikatoren der Bewegungsförderung $(n=19)$.

\begin{tabular}{|c|c|c|c|c|c|}
\hline Bezugsraum & Politik \& Verwaltung & Sport (Non-Profit) & Sport (For-Profit) & Gesundheit & $\begin{array}{l}\text { Bildung \& } \\
\text { Forschung }\end{array}$ \\
\hline Nationale Ebene & $\begin{array}{l}\text { BMG, BZgA, Deutscher } \\
\text { Städtetag, Politiker / Ver- } \\
\text { treter der Politik in der } \\
\text { Öffentlichkeit }\end{array}$ & $\begin{array}{l}\text { DAV, DOSB, DTB, } \\
\text { DWV }\end{array}$ & & Krankenkassen & dvs \\
\hline Landesebene & $\begin{array}{l}\text { Akteure / Ministerien auf } \\
\text { Landesebene }\end{array}$ & LSB / LSV & & & \\
\hline $\begin{array}{l}\text { Regionale / Kom- } \\
\text { munale Ebene }\end{array}$ & Umweltamt, Verkehrsamt & & Fitnessstudios & & $\begin{array}{l}\text { Sportlehrer, Sport- } \\
\text { wissenschaftler / } \\
\text { Institute für Sport- } \\
\text { wissenschaft, Uni- } \\
\text { versität / Hoch- } \\
\text { schule, VHS }\end{array}$ \\
\hline
\end{tabular}

Abkürzungen: DAV: Deutscher Alpenverein, dvs: Deutsche Vereinigung für Sportwissenschaft, DWV: Deutscher Wanderverband, LSB / LSV:

Landessportbünde / Landessportverbände

(Umweltamt, Verkehrsamt), welche in direktem Zusammenhang mit den zuvor genannten Schlüsselpositionen der Kommune sowie der Stadt-, Verkehrs- und Landschaftsplanung stehen. Ebenso wurde die Berufsgruppe der Sportlehrer genannt, welche Teil des Schlüsselakteurs Schule sind. In diesem Zusammenhang sind auch Sportwissenschaftler zu sehen, welche durch die Ausbildung der Sportlehrer und anderer im Bereich der Bewegungsförderung tätiger Berufsgruppen an den Universitäten und Hochschulen eine zentrale Funktion als Multiplikatoren der Bewegungsförderung innehaben. Darüber hinaus wurden Akteure wie Vertreter der Politik in der Öffentlichkeit und verschiedene Sportverbände aufgeführt, die insbesondere durch ihren Einfluss auf Vereine und die Übungsleiterausbildung eine Multiplikatorenrolle einnehmen.

Bezogen auf die Gesellschaftsbereiche werden die Multiplikatoren v. a. in den Bereichen der Politik und Verwaltung, Sport (Non-Profit) und im Bereich der Bildung und Forschung gesehen. Während in den ersten 2 Bereichen die Akteure auf der nationalen Ebene überwiegen, sind es im Bereich der Bildung vor allem auf regionaler und lokaler Ebene tätige Multiplikatoren wie Sportlehrer, Sportwissenschaftler oder die Volkshochschulen. Interessanterweise nannten die Experten keine Multiplikatoren in den Bereichen Familie, Soziales \& Umwelt, Wirtschaft und Medien.

\section{Potenzielle Multiplikatoren}

Auf die Frage nach den Akteuren, die das Potential besitzen, zukünftig als Multiplikator aktiv zu werden, wurden von den Interviewten insgesamt 23 Akteure benannt (vgl. > Tab. 1.4).

Hierbei kam es aufgrund unterschiedlicher Einschätzungen der Experten zu Überschneidungen der potenziellen mit den bereits bestehenden Multiplikatoren. Beispielsweise werden BMG und BZgA von einigen Experten bereits als bestehende Multiplikatoren angesehen, wohingegen andere diese Akteure lediglich als potenzielle Multiplikatoren betrachten. Dies könnte auf vorhandenes Entwicklungspotential bei bereits bestehenden Multiplikatoren hinweisen. Akteure, die ausschließlich als potenzielle Multiplikatoren, und nicht bereits zuvor als Multiplikator genannt wurden, wurden in Tabelle 1.4 mit einem Asterisk $\left({ }^{*}\right)$ versehen. Insbesondere diese Akteure sollten neben den bestehenden Multiplikatoren für eine zukünftige Disseminierung der NEBB berücksichtigt werden.

2 Aspekte fallen hinsichtlich der Verteilung über die Gesellschaftsbereiche auf. Zum einen wurden potenzielle Multiplikatoren auch in den Bereichen Wirtschaft, Familie, Soziales \& Umwelt und Medien genannt. Zum anderen findet sich eine Vielzahl der Multiplikatoren auf der regionalen/kommunalen Ebene wieder. Diese Ebene setzt häufig unmittelbar an den Lebenswelten der Bevölkerung an. Im kommunalen Bereich der Politik und Verwaltung sind dies die kommunalen Gesundheitskonferenzen sowie die Akteure im Bereich der Stadt-, Verkehrs- und Landschaftsplanung. Allerdings wurden auch auf der Landes- und Bundesebene etliche Akteure aus dem Politik- und Verwaltungsbereich angeführt. So wurde z.B. der Bund in diesem Zusammenhang erwähnt, da eine flächendeckende und effektive Informationsverbreitung und Förderung des Themas Bewegungsförderung die Unterstützung von Akteuren der Bundesregierung benötigt.

Im Bereich der Gesundheit ist es offensichtlich, dass die Berufsgruppe der Arbeitsmediziner mit Bezug zum Setting Betrieb eine wichtige Rolle spielen kann, wobei hier auch ein Bezug zum Bereich der Wirtschaft gegeben ist. Interessant ist im Bereich der Gesundheit, dass zwar Krankenkassen im Allgemeinen als wichtiger bestehender Multiplikator genannt wurden, private Krankenkassen aber noch nicht in dieser Rolle gesehen wurden. Diese würden aber einen bedeutsamen potenziellen Multiplikator darstellen, wenn sie sich des Themas stärker annähmen, oder wie ein Experte es ausdrückte: „(...) dann würden die natürlich ein wahnsinniger Transmissionsriemen sein“ (B5, 90).

Im Bereich der Bildung und Forschung wurden neben der dvs auf nationaler Ebene die VHS, Kindergärten, Schulen und Universitäten auf kommunaler Ebene genannt. Diese 4 Akteure decken den Bildungsbereich über die ganze Lebensspanne von der Kindheit bis zum Seniorenalter ab. In diesem Zusammenhang sind auch soziale Einrichtungen und Kirchen zu betrachten. Außerdem 
Tab. 1.4 Potenzielle Multiplikatoren der Bewegungsförderung $(n=23)$.

\begin{tabular}{|c|c|c|c|c|c|c|}
\hline Bezugsraum & $\begin{array}{l}\text { Politik \& } \\
\text { Verwaltung }\end{array}$ & Gesundheit & $\begin{array}{l}\text { Bildung \& } \\
\text { Forschung }\end{array}$ & Wirtschaft & $\begin{array}{l}\text { Familie, Sozia- } \\
\text { les \& Umwelt }\end{array}$ & Medien \\
\hline Nationale Ebene & $\begin{array}{l}\text { Bund*, BMG, } \\
\text { BZgA, Deutscher } \\
\text { Städtetag }\end{array}$ & $\begin{array}{l}\text { Private } \\
\text { Krankenkassen* }\end{array}$ & dvs & $\begin{array}{l}\text { Sportartikel- } \\
\text { hersteller*, } \\
\text { BBGM* }^{*}\end{array}$ & Kirche* & $\begin{array}{l}\text { Medien*, } \\
\text { Schauspieler* }\end{array}$ \\
\hline Landesebene & $\begin{array}{l}\text { Gesunde Städte } \\
\text { Netzwerk*, Kul- } \\
\text { tusministerium*, } \\
\text { Landesgesund- } \\
\text { heitskonferenz* }\end{array}$ & & & & & Medien* \\
\hline $\begin{array}{l}\text { Regionale / Kom- } \\
\text { munale Ebene }\end{array}$ & $\begin{array}{l}\text { Kommunale } \\
\text { Gesundheitskon- } \\
\text { ferenz* , SVLP* }\end{array}$ & $\begin{array}{l}\text { Arbeitsmedizi- } \\
\text { ner* }\end{array}$ & $\begin{array}{l}\text { Kindergarten*, } \\
\text { Schule*, Universi- } \\
\text { tät / Hochschule, } \\
\text { VHS }\end{array}$ & & $\begin{array}{l}\text { Soziale Einrich- } \\
\text { tungen* }\end{array}$ & $\begin{array}{l}\text { Medien*, Otto } \\
\text { Normalverbrau- } \\
\text { cher als Identifi- } \\
\text { kationsfigur* }\end{array}$ \\
\hline
\end{tabular}

wurden etwa Sportartikelhersteller erwähnt, die aufgrund ihrer hohen medialen Präsenz als ein potenzieller Multiplikator betrachtet werden.

Schließlich wurde deutlich, dass den Medien ein großes Potenzial als Multiplikator der Bewegungsförderung auf allen Bezugsebenen zukommt. Dabei muss unterschieden werden zwischen verschiedenen Medienformaten, die Inhalte transportieren, und medialen Akteuren wie Schauspielern, die sowohl in ihren Rollen als auch als in ihrer Vorbildfunktion von Bedeutung sind. Zudem umfasst der Bereich der Medien Kampagnen. In diesem Zusammenhang wurde auch der Durchschnittsbürger oder „Otto Normalverbraucher als Identifikationsfigur“ angeführt, da die Bevölkerung realistische Vorbilder benötigt. Leistungssportler wurden dahingegen kritisch im Zusammenhang mit Kampagnen gesehen, da sie als nicht erreichbare Vorbilder gesehen werden könnten. Nicht zuletzt wird den digitalen Medien eine tragende Rolle als potenzieller Multiplikator hinsichtlich jugend- und subkultureller Zielgruppen zugetraut, die eher auf informellen Wegen erreichbar sind.

Insgesamt 23 identifizierte potenzielle Multiplikatoren weisen auf ein Potenzial hin, welches für eine zukünftige Bewegungsförderung genutzt werden kann. Dabei sind insbesondere die 17 von Interesse, die noch nicht als bestehender Multiplikator berücksichtigt wurden, sowie einzelne Akteure großer gesellschaftlicher Bedeutung wie die erwähnten Medien und der Bund, aber auch Akteure wie die Kirchen oder die privaten Krankenkassen.

\section{Strukturelle Akteursvernetzung}

Im Rahmen der Interviews wurde nach dem Beziehungszusammenhang der durch die Experten genannten Akteure gefragt. Dabei waren zum einen Kooperationsbeziehungen (z. B. Informationsaustausch, informelle Zusammenarbeit, formelle Zusammenarbeit, Partnerschaft) und zum anderen Konkurrenz- oder Konfliktsituationen im Bereich der Bewegungsförderung von Interesse. Dem explorativen Ansatz folgend stand es zunächst im
Vordergrund Kooperations- und Konfliktbeziehungen aller Art zu erfassen, um so einen Gesamtüberblick zu erhalten. Auf Grundlage der Interviewauswertung ließen sich Netzwerkmatrizen erstellen, welche zur Visualisierung der Beziehungszusammenhänge sowie zur Berechnung deskriptiver Netzwerkparameter des bestehenden Netzwerks der Bewegungsförderung in Deutschland dienten. Die erhobenen Beziehungen sind ungerichtet (reziprok) und wurden in 2 Netzwerken getrennt nach Kooperationsbeziehungen und Konflikt-/Konkurrenzbeziehungen ausgewertet. Da die Analyse auf qualitativen Daten und einer selektiven Stichprobe von Experten basierte, wurden Beziehungen binär als entweder vorhanden oder nicht vorhanden behandelt. Beziehungsstärken wurden nicht berücksichtigt, da die Daten darüber keine Rückschlüsse zulassen. Das Ziel der Netzwerkanalyse war, das zum Zeitpunkt der Befragung bestehende Netzwerk so zu rekonstruieren, wie es sich den Experten darstellte. Die verschiedenen Wissensbestände wurden hierbei zusammenfassend in einem Netzwerk dargestellt. Auf diese Weise sind strukturelle Zusammenhänge sichtbar, die über Einzelperspektiven hinausgehen.

Zunächst wurde die globale Netzwerkstruktur betrachtet. Mit 128 Akteuren handelt es sich um ein verhältnismäßig großes Netzwerk, welches gleichzeitig durch wenige Beziehungen (Ties) geprägt ist (vgl. > Tab. 1.5). Im vorliegenden Kooperationsnetzwerk verfügt ein Akteur im Schnitt über 1,125 Kooperationsbeziehungen, womit 0,9\% der möglichen Beziehungen (Density) realisiert wurden. Das Netzwerk besteht aus vielen unverbundenen Teilgruppen (Components) und ist aus diesem Grund stark fragmentiert, wobei $86,6 \%$ der Netzwerkakteure unerreichbar sind. Die durchschnittliche geodätische Distanz zwischen den erreichbaren Akteuren ist mit 3,35 hoch. Das Konflikt-/ Konkurrenznetzwerk ist in noch stärkerem Maße als zusammenhangslos zu bezeichnen, wie die Netzwerkparameter zur Kohäsion zeigen. Der bessere Wert der durchschnittlichen Distanz ist lediglich auf die geringere Anzahl verbundener Akteure zurückzuführen. 
- Tab. 1.5 Globale Netzwerkstrukturen.

\begin{tabular}{|l|l|l|}
\hline Parameter & $\begin{array}{l}\text { Kooperations- } \\
\text { netzwerk }\end{array}$ & $\begin{array}{l}\text { Konflikt- } / \text { Kon- } \\
\text { kurrenznetz- } \\
\text { werk }\end{array}$ \\
\hline Size & 128 & 128 \\
\hline Ties & 144 & 24 \\
\hline Cohesion & & \\
\hline Avg Degree & 1.125 & 0.188 \\
\hline Density & 0.009 & 0.001 \\
\hline Fragmentation & 0.866 & 0.997 \\
\hline Components & 78 & 117 \\
\hline Avg Distance & 3.352 & 1.565 \\
\hline
\end{tabular}

Die Analyse des Gesamtnetzwerkes zeigt, dass nur eine geringe Ausprägung eines Netzwerks zur Bewegungsförderung besteht. Einerseits wurden nur wenige Kooperationsbeziehungen benannt, andererseits sind Konflikte und Konkurrenzsituationen nach Meinung der Experten in noch geringerem Ausmaß gegeben. Hierbei ist zu berücksichtigen, dass die Analyse auf dem Wissen und der Wahrnehmung der Experten beruht und nur die diesen bekannten Beziehungen umfasst.

\section{Kooperationsnetzwerk}

Die Betrachtung der individuellen Netzwerkpositionen der Akteure liefert weitergehende Einsichten in die Kooperationsstrukturen. In \ Tab. $\mathbf{1 . 6}$ sind die 10 aktivsten Akteure aufgrund der Degree-Zentralität dargestellt. Es zeigt sich, dass im Rahmen der Bewegungsförderung v. a. Sportverbände (DTB, DOSB) und Krankenkassen auf der nationalen Ebene die zentralsten Akteure im Kooperationsnetzwerk darstellen. Aber auch der Bund

-Tab.1.6 Individuelle Netzwerkposition (Degree-Zentralität) im Kooperationsnetzwerk (Top 10).

\begin{tabular}{|c|c|c|}
\hline Akteur & Degree & nDegree \\
\hline Deutscher Turnerbund (DTB) & 12 & 0.094 \\
\hline $\begin{array}{l}\text { Deutscher Olympischer Sportbund } \\
\text { (DOSB) }\end{array}$ & 11 & 0.087 \\
\hline Krankenkassen & 9 & 0.071 \\
\hline $\begin{array}{l}\text { Bund deutscher Landschaftsarchitekten } \\
\text { (BDLA) }\end{array}$ & 7 & 0.055 \\
\hline Deutscher Städtetag & 6 & 0.047 \\
\hline Schule & 5 & 0.039 \\
\hline Kultusministerien & 5 & 0.039 \\
\hline $\begin{array}{l}\text { Deutscher Verband für Gesundheits- } \\
\text { sport und Sporttherapie (DVGS) }\end{array}$ & 5 & 0.039 \\
\hline $\begin{array}{l}\text { Bundesverband der Berufsfachschulen } \\
\text { für Gymnastik und Sport (BBGS) }\end{array}$ & 5 & 0.039 \\
\hline $\begin{array}{l}\text { Landessportbund / Landessportverband } \\
\text { (LSB / LSV) }\end{array}$ & 4 & 0.031 \\
\hline
\end{tabular}

deutscher Landschaftsarchitekten (BDLA) und der Deutsche Städtetag stellen verhältnismäßig aktive Akteure dar. Ebenso ist der Bereich der schulischen Ausbildung mit den Schulen, Kultusministerien und dem Bundesverband der Berufsfachschulen für Gymnastik und Sport (BBGS) umfassend vertreten. Ergänzt werden die 10 aktivsten Akteure durch den DVGS. Neben konkreten Kooperationen in der Ausbildung (z. B. zwischen BBGS und DVGS) oder der Förderung von Angeboten (z.B. Krankenkassen und Sportvereine/-verbände), wurden sehr häufig Beziehungen des Informationsaustauschs z. B. in gemeinsam besetzten Arbeitsgruppen genannt. Dies weist auf eher schwach ausgeprägte Kooperationsbeziehungen hin.

In $>$ Abb. 2 ist das Kooperationsnetzwerk der nationalen Bewegungsförderung aus Sicht der Experten als Graph dargestellt. Für eine übersichtlichere Darstellung wurden die 75 unverbundenen Akteure nicht dargestellt. Das Netzwerk besteht so aus 53 Akteuren, die als Knotenpunkte dargestellt sind. Die Größe des Knotens spiegelt die Degree-Zentralität und damit das Maß der Kooperationsaktivität wider. Schlüsselakteure sind in Kreisform dargestellt, alle weiteren Akteure haben die Form eines Quadrats. Die Akteure sind mit Namen (Label) gekennzeichnet, wobei zur übersichtlicheren Darstellung teilweise Abkürzungen verwendet wurden. Ein rotes Label kennzeichnet einen Multiplikator (z. B. DTB). Potenzielle Multiplikatoren sind durch einen roten Rand der Knotenform gekennzeichnet (z. B. Schule). Schließlich wurde ein Layout gewählt, welches die Akteure nach Gesellschaftsbereichen gruppiert. Grüne Akteure entstammen dem Bereich Sport, dunkelblaue Akteure dem Bereich Familie, Soziales \& Umwelt (hier: NABU), graue Akteure dem Bereich Wirtschaft, hellblaue Akteure dem Bereich Gesundheit, gelbe Akteure dem Bereich Politik und Verwaltung und weiße Akteure dem Bereich Bildung und Forschung. Eine Verbindung zwischen den Knotenpunkten stellt eine Kooperationsbeziehung dar.

Die Visualisierung zeigt, dass die zuvor genannten Schlüsselakteure (Kreisform) auch netzwerkanalytisch häufig die zentralsten Akteure sind (z. B. DOSB, DTB, Krankenkassen, Schule, DVGS). Die meisten Multiplikatoren (rotes Label) innerhalb dieses Netzwerks werden im Bereich des Sports gesehen. In den Bereichen Politik und Verwaltung sowie Bildung und Forschung sind die meisten potenziellen Multiplikatoren (roter Rand) verortet. Insgesamt besteht zwischen den Akteuren aus dem Bereich Sport sowie Politik und Verwaltung die stärkste Vernetzung. Der Bereich Bildung und Forschung ist sehr vielfältig in alle Bereiche vernetzt. Ebenso ist der Bereich der Gesundheit durch eine sehr vielfältige Vernetzung gekennzeichnet. Die Bereiche der Wirtschaft sowie Familie, Soziales \& Umwelt sind dagegen mit nur sehr wenigen Akteuren vertreten und in der Folge nur schwach in das Kooperationsnetzwerk der Bewegungsförderung eingebunden.

\section{Konflikt- und Konkurrenznetzwerk}

Die strukturelle Analyse der bestehenden Konflikt- und Konkurrenzsituationen in der Bewegungsförderung deutet auf ein nur in Ansätzen bestehendes Netzwerk hin, welches aus insgesamt 15 Akteuren besteht. Aufgrund der geringen Substanz wird auf eine differenzierte Darstellung der Zentralitätswerte der 15 Akteure (Min.=1; Max.=3) sowie eine Visualisierung des 


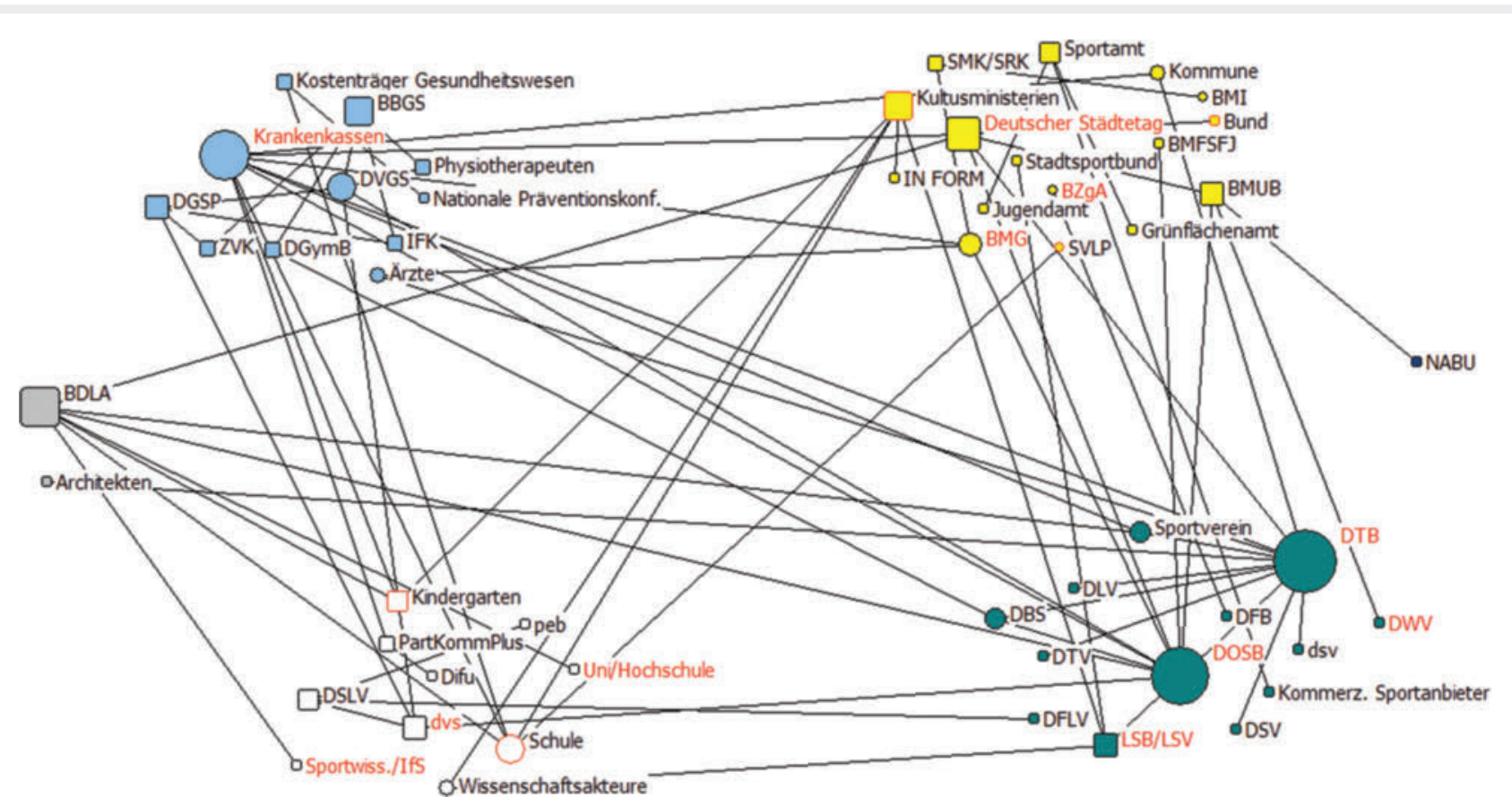

Abb.2 Kooperationsstrukturen in der Bewegungsförderung ( $n=53)$.

Netzwerks verzichtet. Die geringe Substanz kann zum einen als positiv erachtet werden, da die Experten nur wenige Konflikte sehen, zum anderen ist dies auch eine Folge der insgesamt geringen Ausprägung der Netzwerkstrukturen. Hinsichtlich der Konflikte und Konkurrenzsituationen finden sich der DOSB, die Krankenkassen und die Sportvereine in den zentralsten Positionen. Die Sportvereine werden im Rahmen der Bewegungsförderung beispielsweise in einer Konkurrenzsituation mit den Fitnessstudios um Mitglieder bzw. Kunden betrachtet. Ebenso wird ein Konflikt zwischen Vertretern des organisierten Sports (DOSB bzw. Sportvereine) und Akteuren eines informellen, nicht-organisierten Sports gesehen, da die meiste Sportaktivität im Sinne einer Bewegungsförderung nicht im organisierten Sport, sondern informell organisiert stattfindet. Ein weiteres Beispiel für eine Konkurrenzsituation ist die Beziehung zwischen DOSB und DVGS in Bezug auf eine nicht-berufliche Ausbildung (DOSB) im Gegensatz zu einer professionellen Ausbildung von Bewegungsförderern (DVGS). Ein möglicher Interessenskonflikt wurde auch zwischen Krankenkassen und der Berufsgruppe der Ärzte ausgemacht. So kann die Situation entstehen, dass die Krankenkassen aus wirtschaftlichen Gründen ein Interesse daran haben, dass die Menschen präventiv Sport treiben und so potenziell eine ärztliche Behandlung nicht benötigen. Dagegen haben Ärzte möglicherweise ein geringeres Interesse an Bewegungsförderung, da es sich für sie derzeit um eine Maßnahme ohne direkten wirtschaftlichen Nutzen handelt.

Die Gesamtbetrachtung des Konflikt- und Konkurrenznetzwerks verdeutlicht, dass Konkurrenzsituationen in der Bewegungsförderung am häufigsten zwischen Akteuren aus dem Sport (DOSB, Sportvereine) und Vertretern des Gesundheitsbereichs (DVGS, Bundesverband selbstständiger Physiotherapeuten,
Zentralverband Physiotherapeuten, Berufsgruppe der Physiotherapeuten) gesehen werden.

\section{Netzwerkentwicklung}

Mit Blick auf eine zukünftige Entwicklung eines nationalen Netzwerkes der Bewegungsförderung ist es notwendig, den Nutzen bzw. die Vorteile für die Beteiligten sowie mögliche Barrieren bzw. Nachteile zu identifizieren. Aus diesem Grund wurden die Experten nach ihrer Einschätzung in dieser Hinsicht gefragt.

\section{Voraussetzungen einer Netzwerkentwicklung}

Zunächst wurden verschiedene Voraussetzungen genannt, die zur Bildung eines effektiven und dauerhaften Netzwerks notwendig sind.

Als entscheidend für die Gründung und den 1. Anschub eines Netzwerks wurde die Kommunikation auf politischer Ebene angeführt:

„Ich glaube erst einmal, dass man das dann politisch geschickt kommunizieren muss, weil ich glaube, auf politischer Ebene ist es gar nicht klar oder sind die Auswirkungen, der momentane Zustand, gar nicht bewusst." $(B 10,135)$

Voraussetzung ist demzufolge, dass die Thematik einer Bildung eines nationalen Netzwerks der Bewegungsförderung zunächst auf die politische Agenda gelangen muss. Erst durch eine politische Diskussion könnte die Relevanz der Thematik die notwendige politische Bedeutung damit die notwendige Unterstützung und Anerkennung erfahren, die für eine erfolgreiche Netzwerkbildung notwendig wäre.

Damit hängt die Einschätzung eines Experten zusammen, wonach es neben einer politischen Willensbildung zur Netzwerkentwicklung 
einen konkreten politischen Beschluss zur Durchsetzung der Netzwerkentwicklung geben müsste, der auch die Landesregierungen zum Handeln auffordert.

„Also es müsste ein Beschluss der Bundesregierung sein. Also da reicht auch (...) das BMG alleine nicht aus. (...) das müsste zum Beispiel im Koalitionsvertrag stehen. Es müsste dann eben auch wirklich dafür geworben werden, dass sich die Bundesländer aus eigenem Interesse daran beteiligen. “ $(B 8,150)$

In diesem Zusammenhang wurde der Einwand formuliert, dass eine rein nationale Strategie aufgrund der Unterschiede der Bundesländer scheitern könnte. Aus diesem Grund wäre eine TopDown-Strategie notwendig, im Rahmen derer von der nationalen Ebene ausgehend gezielt die Landes- und Kommunalebenen mit eingebunden werden. Demnach bedarf eine nationale Strategie der Netzwerkentwicklung auch der Unterstützung und Verwirklichung auf kommunaler Ebene. Ein möglicher Weg, der in diesem Zusammenhang vom Experten genannt wurde ist, dass von der Bundesregierung ausgehend eine Vernetzung über die Landesregierungen bzw. den Deutschen Städtetag bis in die Kommunen stattfindet.

Nach Meinung einer Expertin könnte die Bildung settingbezogener Netzwerke mit dem Fokus auf einen intensiven Austausch über konkrete Themenfelder wie etwa „Bewegungsförderung in Pflegeheimen“ (B2, 86) notwendig sein, damit ein Netzwerk der Bewegungsförderung funktioniert. Auf diese Weise entstünden verschiedene Netzwerke, die sich in ihrer settingspezifischen Arbeit gegenseitig befruchten könnten.

Damit die Netzwerkarbeit effektiv ist und dadurch nicht unnötig personelle und finanzielle Ressourcen verschwendet werden, wurden zudem die Notwendigkeit einer konkreten Zielformulierung sowie einer zeitlichen Strukturierung im Sinne eines effizienten Zeitmanagements angesprochen.

Zusammenfassend wird einerseits die Notwendigkeit eines starken politischen Willens oder gar Beschlusses auf Bundesebene als Voraussetzung für die Bildung eines nationalen Netzwerks der Bewegungsförderung gesehen. Andererseits wird auch eine Netzwerkbildung auf kommunaler Ebene oder bezogen auf spezifische Settings als wichtig erachtet. Dies würde einer TopDown-Strategie der Netzwerkentwicklung entsprechen. Zudem werden eine konkrete Zielformulierung und effizientes Zeitmanagement als Voraussetzungen einer erfolgreichen Netzwerkbildung betrachtet $(\triangleright$ Tab. 1.7$)$.

\section{Nutzen einer Netzwerkbeteiligung}

Die Vorteile einer Netzwerkentwicklung bzw. aktiven Beteiligung sind aus Sicht der Befragten vielseitig. Sie beziehen sich zum einen auf nützliche Wirkungen und Konsequenzen des gesamten Netzwerks, zum anderen aber auch auf den Nutzen für den einzelnen Akteur durch seine Beteiligung am Netzwerk.

Zunächst wird ein Nutzen darin gesehen, auf gesellschaftlicher Ebene Aufmerksamkeit und Einfluss zu gewinnen:

„Der größte Nutzen eines solchen Netzwerks bestünde darin, dass das Thema Sport und Bewegung als Gesundheitsfaktor dann in
-Tab. 1.7 Voraussetzungen einer Netzwerkentwicklung.

Kommunikation auf politischer Ebene (Agenda-Setting)

Politische Willensbildung und / oder Beschluss einer

Netzwerkentwicklung

Unterstützung und Verwirklichung der Netzwerkbildung auf kommunaler Ebene (Top-Down-Strategie)

Bildung ausdifferenzierter, settingbezogener Netzwerke

Konkrete Formulierung von Netzwerkzielen

Effizientes Zeitmanagement der Netzwerkarbeit

vielen unterschiedlichen Gesellschaften sozusagen zum einen erstmal bekannt ist und man über diese Netzwerke dann dieses Thema auch voranbringen kann und etwas bewegen kann." $(B 5,105)$

In ähnlicher Weise wird der Nutzen eines solchen Netzwerks in der gesteigerten Möglichkeit gesehen, Einfluss auf eine große Zielgruppe zu nehmen. Hierbei wird vor allem ein verstärkter gesellschaftlicher Einfluss erwartet, der durch ein konzentriertes, gemeinsames Vorgehen verschiedener in dem Netzwerk beteiligter Multiplikatoren entstünde. Auf der anderen Seite wird eine Netzwerkbildung als Zusammenschluss einer Interessensvertretung mit dem konkreten Nutzen einer Machtbündelung und verbesserten Einflussnahme auf politischer Ebene betrachtet.

In diesem Zusammenhang wurde darauf verwiesen, dass durch eine Steigerung der Bedeutsamkeit und stärkeren öffentlichen Aufmerksamkeit, die ein solches Netzwerk erwarten lässt, eine erhöhte Bereitstellung von Ressourcen auf politischer Ebene eingeleitet werden kann. Die Verfügbarkeit dieser Ressourcen wird als ein bedingender Faktor betrachtet.

„Anreize auf Landesebene zum Beispiel: Wenn ich diesem Thema in der Kommune mehr Aufmerksamkeit gebe oder geben möchte, brauche ich Ressourcen, die dann eben auch personell ausgestattet sein müssen, um diesem Thema Rechnung zu tragen. Das heißt dann auch Ressourcenverschiebung oder zumindest Allokation auf Landes- und kommunaler Ebene in dieses Themenfeld. Das heißt, es braucht auch wieder Geld an der Stelle. " (B7, 167)

Zudem sieht eine Expertin in der Schaffung eines solchen Netzwerks die Möglichkeit einer weniger einseitigen Interessensvertretung des Sports durch den organisierten Sport. Der Nutzen läge dann auf Seiten einer stärkeren Berücksichtigung der Interessen des informell organisierten Sports, da der informelle Sport bislang noch nicht über eine entsprechende Lobby verfügt.

„(...) das ist natürlich immer ein ziemliches Lobbying, was da auch gemacht wird, also als Alleinvertretung des Sports in Deutschland [gemeint ist hier die Rolle des DOSB] und es ist einfach nicht mehr so. Das zeigen auch die BISp-Studien einfach sehr gut, dass der nicht-organisierte Sport der wahre Träger des Sports (...) ist. Sie haben im informellen Bereich, sei es Joggen, 
Schwimmen, Wandern etc., eigentlich viel mehr [Aktive] als im organisierten Vereinssport.“ $(B 3,287)$

Die bislang diskutierten Vorteile einer Netzwerkentwicklung für Bewegungsförderung setzen v. a. auf der kollektiven Ebene an und beschreiben den Nutzen für die Gemeinschaft. Die Experten thematisierten allerdings auch den Aspekt, dass darüber hinaus der einzelne Akteur einen individuellen Nutzen einer Netzwerkbeteiligung sehen muss, um sich in einem solchen Netzwerk zu engagieren. So gäbe ein Netzwerk Raum für die Initiierung und Umsetzung eigener Ideen in einem geschützten Rahmen und ermöglicht Partizipation als Mittel der Einflussnahme. Dies gilt v. a. für kleinere Akteure mit einem vergleichsweise geringen Maß an politischer Macht und Einfluss, die sich in einem solchen Netzwerk finden und zusammenarbeiten könnten. Zudem liegt ein Nutzen in der vereinfachten Umsetzung eigener Ideen und Projekte durch Partnerschaften, die sich im Rahmen eines solchen Netzwerks bilden können: „Und umgekehrt ist es eben so, dass man über das Netzwerk eben auch Partner finden kann, mit denen man was zusammen machen kann, mit denen man was auf den Weg bringen kann“ (B5, 111).

Ein weiterer wichtiger Aspekt ist Sicherheit durch Informationsaustausch und den Zugang zu Wissen, den ein solches Netzwerk den Akteuren bietet:

B4: „Sicherheit im Sinne dessen, dass ich ja sehr viele Informationen bekomme, auf die ich sonst gar keinen Zugriff hätte. Das heißt, ich bin mit meinen zukünftigen Schritten, die ich gehe, sozusagen ein Stück weit sicherer, weil ich es besser abwägen kann, in welche Richtung ich mich bewege. Ich weiß dann einfach mehr. Also, eine Frage von Bildung letztlich.“

\section{I: „Zugang zu Wissen?“}

B4: „Ja, Zugang zu Wissen und dann entsprechend Sicherheit für zukünftige Entscheidungen, weil man einfach mehr Kenntnisse hat." (B4, 140 - 142)

Insgesamt ergibt sich auf Grundlage der Befragung der Experten ein vielfältiger Nutzen. Sowohl auf Netzwerkebene werden verschiedene Vorteile durch eine Netzwerkbildung erwartet als auch für den einzelnen Akteur, der sich in einem solchen Netzwerk agiert und für seinen Aufwand einen Nutzen erwartet ( Tab. 1.8).

\section{Barrieren der Netzwerkbeteiligung}

Als eine zentrale Barriere wurde die Netzwerkgröße angesprochen. Für eine konkretere Bearbeitung von Themenbereichen riet ein Experte von einem großen und übergreifenden Netzwerk ab. Mehrere kleine Netzwerke, die sich auf die wesentlichen Dinge konzentrieren können, sind demzufolge vorteilhafter für die Bearbeitung von (Teil-)Aufgaben: „Und dann halte ich es für besser, man macht nicht ein großes Netzwerk, sondern man macht vielleicht viele kleine Netzwerke, wo ganz konkrete Dinge angegangen werden.“ (B6, 202) Die geäußerten Bedenken entsprechen den genannten Voraussetzungen, bei denen die Bildung ausdifferenzierter, settingbezogener Netzwerke bzw. kommunaler Netzwerke, welche eher kleiner und spezifischer angelegt sind, angeregt wurde.
- Tab. 1.8 Übersicht zum Nutzen einer Netzwerkentwicklung / -beteiligung.

\section{Nutzen einer Netzwerkentwicklung insgesamt}

Zugewinn von Aufmerksamkeit und Einfluss für Bewegungsförderung auf gesellschaftlicher Ebene

Einflussnahme auf politischer Ebene / Machtbündelung

Bereitstellung von Ressourcen auf politischer Ebene

Interessensausgleich / weniger einseitige Interessensvertretung des Sports durch den organisierten Sport

\section{Nutzen einer Netzwerkbeteiligung für den einzelnen Akteur}

Initiierung und Umsetzung eigener Ideen

Partizipation / Möglichkeit der individuellen Einflussnahme

Bildung von Partnerschaften

Sicherheit durch Informationsaustausch und den Zugang zu Wissen

Ein anderer nachteiliger Aspekt, der mit der Größe des Netzwerks zusammenhängt, sind langwierige Entscheidungsfindungsprozesse, eine erhöhte zeitliche Beanspruchung und der Mangel an zeitlichen und personellen Ressourcen für die zusätzliche Arbeit im Rahmen des Netzwerks, wie durch verschiedene Experten ausgeführt wurde. Daneben wurde das sogenannte Trittbrettfahrerproblem als ein Hindernis aufgeführt. Ein Interviewpartner bemerkte hierzu knapp: „Wenn es um Arbeit geht, drücken sich die meisten." (B6, 206)

Neben den beschriebenen Barrieren ist eine vorhandene oder sich entwickelnde Konkurrenz- und Konfliktsituation aufgrund kommerzieller Interessen möglich. Ein Experte erläuterte diesen Umstand sowie einen daraus entstehenden Mangel an Vertrauen, der ein Hindernis für eine offene Netzwerkarbeit darstellen kann folgendermaßen:

„Konflikte oder Konkurrenz (...) in Weiterbildungsstrukturen oder dergleichen. Es muss natürlich jeder auch diese Konkurrenzsituationen ein Stück weit überwinden, wenn er in Netzwerken zusammenarbeiten möchte und Informationsaustausch gewährleisten für die Sache. (....) Sobald es irgendwo um kommerzielle Interessen geht, geht es halt nicht nur um die Sache. Und dort kann Informationsaustausch eben auch hinderlich oder als nicht förderlich gesehen werden für den einzelnen Akteur. Oder dass man halt eine bestimmte Partnerschaft, meinetwegen mit Kostenträgern oder dergleichen, auch als privilegiertes Mittel zu nutzen versucht (...). Ich glaube, wenn wir jetzt kurz zurückgehen, als ich über Konkurrenz und Konflikte gesprochen habe, dort gilt es für die Sache zu denken (...) und da könnten Interessensprobleme auftreten. Da bin ich mir nicht so sicher, ob da jeder ganz mit offenen Karten spielen möchte.“ (B7, 175)

Eine allgemeine Barriere, unabhängig von Netzwerkgröße oder Ausrichtung, ist eine fehlende konkrete Zielsetzung für die Netzwerkaktivität, wie ein Experte betonte. So wäre seiner Ansicht nach eine konkrete Zielsetzung und befristete Laufzeit eines 
Netzwerks der Bewegungsförderung notwendig, wobei er die Bewegungsempfehlungen nicht als ausreichende bzw. geeignete Zielsetzung betrachtet:

„Aber diese Netzwerke funktionieren immer nur dann, wenn ein ganz konkretes Ziel dahintersteckt. Ein ganz konkreter Anlass. Und man darf sie auch nicht zu lange laufen lassen, sondern die können eigentlich nur projektbezogen realisiert werden. Ansonsten bringt das gar nichts. Und auf der Grundlage dieser Bewegungsempfehlungen (...) in ein Netzwerk einzusteigen? Nein!“ (B6, 200)

In diesem Zusammenhang ist auch folgende Aussage zu sehen, in der die Interviewpartnerin ausdrückt, dass den Beteiligten deutlich werden muss, inwieweit ein solches Netzwerk Nutzen bringt:

„Netzwerke laufen ja nicht von allein, sondern wenn man Netzwerke ins Leben rufen will oder am Laufen halten will, dann fordert das Energie von den Beteiligten. Und sei es, dass ich da regelmäßig hinfahren muss. Nach meiner Einschätzung ist die Gesundheitsförderung im Moment sehr gesättigt mit Netzwerktreffen in diesem und jenem Bereich. Wenn da ein Gewinn nicht spürbar ist, dann ist die große Gefahr, dass das irgendwie schnell wieder einschläft. “(B2, 98)

Neben einer konkreten Zielsetzung sollte also der Nutzen für die Beteiligten klar und transparent sein. Wie es aussehen kann, wenn das nicht der Fall ist, betonte die Expertin kurz darauf. Eine Vielzahl bereits bestehender Netzwerktreffen mit sich teilweise überschneidenden Zielsetzungen führen ihrer Erfahrung nach zu einer Ablehnung weiterer Netzwerkbeteiligungen.

Die letzten 2 Aspekte (Zielsetzung und Nutzen) korrespondieren mit den bereits bei den Voraussetzungen genannten Aspekten einer konkreten Formulierung von Netzwerkzielen und einem effektiven Zeitmanagement der Netzwerkarbeit. Zusammenfassend bestehen verschiedene Barrieren einer Netzwerkbeteiligung, die im Rahmen einer Netzwerkentwicklung beachtet werden sollten ( $\triangleright$ Tab. 1.9).

\section{Diskussion und Handlungsempfehlungen}

Das erste Ziel der Untersuchung war, die Frage zu beantworten, wer die relevanten Akteure, die Akteure in Schlüsselpositionen, und die aktuellen sowie potenziellen Multiplikatoren der Bewegungsförderung in Deutschland sind.

- Tab. 1.9 Übersicht zu Barrieren der Netzwerkbeteiligung.

Netzwerkgröße

Langwierige Entscheidungsfindungsprozesse

Erhöhte zeitliche Beanspruchung / Mangel an zeitlichen und personellen Ressourcen

Trittbrettfahrerproblem

Konkurrenz- und Konfliktsituation aufgrund kommerzieller Interessen

Fehlende Zielsetzung

Kein klar erkennbarer Nutzen der Netzwerkbeteiligung 
Zunächst zeigte sich, dass das Verständnis relevanter Akteure, Berufsgruppen und Multiplikatoren ausgesprochen heterogen ist, weshalb eine Differenzierung in individuelle und kollektive Akteure, Akteursaggregate, Berufsgruppen und soziale Institutionen vorgenommen wurde, um die Bandbreite aller Akteure der Bewegungsförderung zu erfassen. Insgesamt konnten 128 Akteure aus den Gesellschaftsbereichen Bildung und Forschung, Familie, Soziales und Umwelt, Gesundheit, Medien, Politik und Verwaltung sowie Sport identifiziert werden. Die meisten der relevanten Akteure (33\%) sind dem Bereich Politik und Verwaltung zuzuordnen. Demzufolge ist das Themenfeld Bewegungsförderung in Deutschland primär dem Bereich der Politik und Verwaltung zuzuordnen. Auch wenn Akteure aus anderen Bereichen stark vertreten sind, liegt die entscheidende Rolle in der Umsetzung nachhaltiger Bewegungsförderung bei den politischadministrativen Akteuren des Landes [21]. Dieser Fokus entspricht auch einer Entwicklung in der Gesundheits- und Bewegungsförderung, wonach seit einiger Zeit neben individuumsund zielgruppenbezogenen Ansätzen, die auf das Verhalten abzielen, v. a. politik- und lebensweltbezogene Ansätze, welche durch politisch-administrative Entscheidungen (bspw. in der Städtebau- oder Verkehrspolitik) auf die Verhältnisse abzielen, als effektive und effiziente Ansätze der Bewegungsförderung betrachtet werden [16]. In der Gesamtschau kann man von der Bewegungsförderung als einem von Interdisziplinarität geprägtem Feld sprechen, dass seinen Schwerpunkt im Bereich der Politik und Verwaltung hat.

Bei den am häufigsten genannten relevanten Akteuren steht mit dem DOSB jedoch ein Akteur aus dem Sport an 1. Stelle. Dies verwundert nicht, ist der DOSB nicht zuletzt aufgrund der hohen Mitgliederzahl der angeschlossenen Verbände und Vereine die stärkste nationale Interessensvertretung der Belange des Sports und damit einer der wichtigsten Ansprechpartner für Akteure des Bereichs Politik und Verwaltung. Folglich wurde der DOSB auch als einer der Akteure in einer Schlüsselposition genannt. Daneben fällt bei den Schlüsselpositionen auf, dass hier viele spezifische, individuelle Akteure wie die BZgA, der Bundesgesundheitsminister oder der DVGS angeführt wurden. Das Gros der Akteure befindet sich dabei im Bereich der Politik und Verwaltung auf der nationalen Ebene bzw. der gesellschaftlichen Makroebene. Neben den individuellen Akteuren werden auch Berufsgruppen wie Ärzte und kollektive Akteure wie die Arbeitgeber/der Betrieb genannt, ebenso wie die Familie oder Kommunen als soziale Institutionen. Die 3 letztgenannten Akteure stellen gleichsam lebensweltliche Settings dar, die im Rahmen einer Bewegungsförderung eine Schlüsselposition innehaben und im Rahmen einer Strukturentwicklung berücksichtigt werden sollten.

Für eine Disseminierung von Bewegungsempfehlungen sind insbesondere Multiplikatoren von Bedeutung. Hierbei wurde zwischen bestehenden Multiplikatoren und potenziellen Multiplikatoren der Bewegungsförderung unterschieden. Bei den bestehenden Multiplikatoren waren zahlreiche Akteure politische und verwaltende Organisationen auf der Bundesebene. Zudem wurden viele der genannten Multiplikatoren bereits als Schlüsselakteure genannt (z.B. Krankenkassen, DOSB, BMG). Auffällig ist, dass die Experten bei den bestehenden Multiplikatoren keine Akteure aus den Bereichen Wirtschaft, Familie, Soziales \& Umwelt und Medien sahen.
Hier besteht aufgrund einer offenbar defizitären strukturellen Einbindung von Akteuren dieser Bereiche möglicherweise Entwicklungspotenzial.

Darauf weisen auch die Antworten bezüglich potenzieller Multiplikatoren hin. So wurden etwa Sportartikelhersteller aus dem Bereich der Wirtschaft, die Kirche und soziale Einrichtungen aus dem Bereich Familie, Soziales und Umwelt sowie Medien im allgemeinen, Schauspieler und der „Otto Normalverbraucher als Identifikationsfigur" in der medialen Darstellung als potenzielle Multiplikatoren genannt, die bei einer Disseminierung von Richtlinien und Empfehlungen bezüglich einer Bewegungsförderung zukünftig beachtet werden sollten. Insgesamt konnten im Rahmen der Experteninterviews 17 weitere Akteure identifiziert werden, die bislang keine Funktion als Multiplikator innehaben, dafür nach Meinung der Experten aber das Potenzial besäßen.

In einem weiteren Schritt wurden in einer Übersicht der wichtigsten Akteure der Bewegungsförderung in Deutschland zu allen Akteuren in Schlüsselpositionen sowie zu allen Multiplikatoren und potenziellen Multiplikatoren beschreibende Steckbriefe erstellt (deren Darstellung über den hier zur Verfügung stehen Rahmen hinausgehen würde). Aus diesen geht neben einer Kategorisierung Funktion und Bezug zur Bewegungsförderung, der primäre Gesellschaftsbereich, die Zielgruppe, mögliche Ansprechpartner und das Vorkommen in den Experteninterviews hervor.

Insgesamt wird so eine umfassende Darstellung der relevanten Akteure, Berufsgruppen und Multiplikatoren möglich, welche gleichsam die in Frage kommende Population für die Entwicklung eines flächendeckenden Netzwerks der Bewegungsförderung darstellen.

Das 2. Ziel war es herauszufinden, wie die identifizierten Akteure der Bewegungsförderung in Deutschland miteinander vernetzt sind und welche Bedingungen für eine Netzwerkentwicklung förderlich sind. Daraus lassen sich im Sinne eines Anwendungsbezuges Empfehlungen zur nachhaltigen Entwicklung eines nationalen Netzwerks der Bewegungsförderung erarbeiten.

Auf Grundlage der Experteninterviews konnten 2 Netzwerke konstruiert werden. Ein Netzwerk stellt die Kooperationsstrukturen dar, das andere die Konflikt- und Konkurrenzstrukturen. Insgesamt erwiesen sich die so erstellten Netzwerke zwar als groß, aber auch als stark fragmentiert und unzusammenhängend. Alle Kohäsionsparameter weisen auf einen schwach ausgeprägten Beziehungszusammenhang hin. Dies gilt insbesondere für das sich nur rudimentär abzeichnende Konflikt- und Konkurrenznetzwerk. Die erhobenen Netzwerkdaten verweisen auf ein erhebliches Potenzial der Netzwerkentwicklung, welche zunächst in einer Einbindung der zahlreichen isolierten Akteure bestehen könnte.

In Bezug auf einzelne Akteure lässt sich feststellen, dass der DTB, der DOSB und die Krankenkassen zu den aktivsten Akteuren gehören. Gleichzeitig befinden sich der DOSB und die Krankenkassen zusammen mit den Sportvereinen am häufigsten in Konflikt- oder Konkurrenzsituationen innerhalb dieses Netzwerks. Alle 4 Akteure wurden i. Ü. auch zuvor als Akteure in Schlüsselpositionen bezeichnet. Weitere Schlüsselakteure, die sich auch netzwerkanalytisch in zentraler Position des Kooperationsnetzwerks befinden, sind die Schulen und der DVGS.Die meisten Multiplikatoren sowie potenzielle Multiplikatoren im 
Kooperationsnetzwerk entstammen den Bereichen Sport, Politik und Verwaltung sowie Bildung und Forschung. Akteure der Bereiche Familie, Soziales \& Umwelt, Wirtschaft und Medien sind dagegen nur schwach vertreten und sollten bei einer Netzwerkentwicklung in besonderem Maße berücksichtigt werden.

Im Hinblick auf eine Netzwerkentwicklung konnten im Rahmen der Analyse weitere, zu berücksichtigende Aspekte herausgearbeitet werden. Diese gliedern sich in die Bereiche Voraussetzungen, Nutzen einer Netzwerkentwicklung insgesamt, Nutzen für den einzelnen Akteur und Barrieren einer Netzwerkbeteiligung. Als Voraussetzungen wurden die Kommunikation auf politischer Ebene (Agenda-Setting), die politische Willensbildung und / oder Beschluss einer Netzwerkentwicklung sowie die Unterstützung und Verwirklichung der Netzwerkbildung auf kommunaler Ebene (Top-DownStrategie) genannt. Entscheidend ist hierbei eine Abstimmung und Koordinierung der Bemühungen auf den verschiedenen Ebenen [10, 21]. Weitere Voraussetzungen der Netzwerkentwicklung, die identifiziert wurden, sind die konkrete Formulierung von Netzwerkzielen und ein effektives Zeitmanagement der Netzwerkarbeit. Zuletzt wurde angeregt, dass die Bildung ausdifferenzierter und settingbezogener Netzwerke ein Erfolgsfaktor sein könnte.

Der Nutzen einer Netzwerkentwicklung wurde insgesamt in 4 Bereichen gesehen: 1. würde ein Zugewinn von Aufmerksamkeit und Einfluss für Bewegungsförderung auf gesellschaftlicher Ebene eintreten. 2. wäre eine Einflussnahme auf politischer Ebene im Sinne einer Machtbündelung möglich. 3. würde diese die Bereitstellung von Ressourcen auf politischer Ebene vereinfachen. 4. hätte ein solches Netzwerk den Vorteil eines Interessensausgleichs hinsichtlich der Bewegungsförderung, da die Interessen des Sports weniger einseitig durch den bisher dominierenden organsierten Sport vertreten würden.

Auch für den einzelnen Akteur würde sich durch eine Beteiligung an solch einem Netzwerk ein Nutzen ergeben. Dieser läge in der Möglichkeit einer Initiierung und Umsetzung eigener Ideen, der Partizipation und Möglichkeit der individuellen Einflussnahme, der Bildung von synergetischen Partnerschaften sowie ein Zugewinn an Sicherheit durch Informationsaustausch und den Zugang zu Wissen.

Schließlich wurden verschiedene Barrieren genannt, die im Zuge einer Netzwerkentwicklung berücksichtigt werden sollten. Diese liegen zum einen in der Netzwerkgröße begründet, die ein Arbeiten möglicherweise erschwert und zu langwierigen Entscheidungsprozessen führt. Weitere Barrieren sind eine erhöhte zeitliche Beanspruchung/Mangel an zeitlichen und personellen Ressourcen, das Problem von „Trittbrettfahrern“, möglicherweise entstehende Konkurrenz- und Konfliktsituation aufgrund kommerzieller Interessen, eine fehlende Zielsetzung sowie kein klar erkennbarer Nutzen der Netzwerkbeteiligung.

Bezugnehmend auf Erkenntnisse aus der Forschung zur Netzwerkgovernance [13] lassen sich folgende Schlüsse zur Steuerung des vorliegenden Netzwerks ziehen. Es handelt es sich um ein relativ großes Netzwerk, das weniger zur Bearbeitung spezifischer Problemstellungen geeignet ist, sondern eher einer Koordination gemeinsamer Interessen dienlich ist. Spezifische Probleme sollten in kleineren Arbeitsgruppen bzw. sich selbst steuernden Netzwerken, die z. B. settingbezogen ausgerichtet sind, bearbeitet werden. Hierbei ist eine klare Zielsetzung (und ggf. zeitliche Begrenzung) die Voraussetzung für eine effektive Netzwerkarbeit. Aufgrund der Größe und der geringen Dichte ist für eine effiziente Netzwerksteuerung die Einrichtung einer administrativen Einheit (Network Administrative Organisation) notwendig, die unabhängig von den beteiligten Akteuren agiert. Diese wird von den beteiligten Akteuren kontrolliert, sollte keine kommerziellen Interessen haben und ist eine Bedingung für die Entwicklung von Vertrauen in das Netzwerk. Zudem könnten so vor- und nachbereitende Arbeiten für Netzwerktreffen sowie Koordinationsaufgaben im Sinne eines effektiven Netzwerkmanagements übernommen werden. Diese koordinierende Organisation müsste im Allgemeinen über ein hohes Maß an Kompetenzen der Netzwerksteuerung verfügen. Nicht zuletzt ist ein entscheidender Faktor für eine effektive Netzwerksteuerung die Verständigung über ein gemeinsames Ziel, welches die Voraussetzung für eine strategische Vorgehensweise darstellt. In diesem Zusammenhang ist zu erwähnen, dass die WHO in ähnlicher Weise die Einrichtung einer Führungsstelle auf oberster Regierungsebene sowie die Entwicklung von Koordinierungsmechanismen zwischen den verschiedenen gesellschaftlichen Akteuren fordert, um gemeinsame Ziele und Synergien zu entwickeln. Zur Sicherstellung der Effektivität bedarf die Koordinierung demnach einer klaren Rollenverteilung, einer Ressourcenbereitstellung und Evaluation [21]. Die angesprochenen Aspekte der Netzwerksteuerung sind entscheidend für die erfolgreiche Umsetzung einer nationalen Bewegungsförderung. Wie Beispiele aus anderen Ländern zeigen, stellen mangelnde Koordination, das Fehlen eines strategischen Plans sowie eine fehlende Übernahme von Verantwortung durch Beteiligte gravierende Defizite bei der Umsetzung nationaler Pläne zur Bewegungsförderung dar [2, 18].

Zusammenfassend ist ein in der Breite vorhandenes, aber strukturell defizitäres Netzwerk einer Bewegungsförderung zu erkennen. Hinsichtlich einer Einbettung isolierter, aber für die Bewegungsförderung relevanter Akteure besteht ein hohes Entwicklungspotenzial. Darüber hinaus lassen sich verschiedene Faktoren einer nachhaltigen Netzwerkentwicklung aufzeigen.

Abschließend lassen sich folgende Handlungsempfehlungen aussprechen, die für die Entwicklung eines nachhaltigen Netzwerks für Bewegungsförderung dienlich sind und eine flächendeckende Dissemination der NEBB unterstützen können.

- Um das Ziel einer flächendeckend vernetzten Bewegungsförderung zu erreichen, ist es notwendig, das weitreichende aber stark fragmentierte und schwach ausgeprägte Netzwerk von relevanten Akteuren der Bewegungsförderung zu stärken. Hierbei sind insbesondere die identifizierten Schlüsselakteure und Multiplikatoren zu berücksichtigen.

- Da die Gruppe relevanter Akteure relativ groß sowie heterogen ist und sich über viele Gesellschaftsbereiche erstreckt, bedarf es eines koordinierten Ansatzes in dessen Rahmen eine klare Zielsetzung des Netzwerks und Verantwortlichkeiten formuliert werden.

- Neben dem Ziel muss sowohl der übergeordnete Nutzen des Netzwerks (z. B. Zugewinn von Aufmerksamkeit und Einfluss für Bewegungsförderung, politische Einflussnahme im Sinne einer Machtbündelung, Bereitstellung von Ressourcen auf politischer Ebene, Interessensausgleich) als auch für die Beteiligung des einzelnen Akteurs (z. B. Initiierung und Umsetzung eigener 
Ideen, Möglichkeit der individuellen Einflussnahme, Bildung von Partnerschaften, Zugang zu Wissen) deutlich werden.

- Neben der Integration isolierter Akteure sollte eine vorrangige Aufgabe die vermehrte Integration von Akteuren aus den Bereichen Familie, Soziales \& Umwelt, Wirtschaft sowie Medien in das Netzwerk sein. Diese können eine wichtige Funktion als Multiplikatoren neben den bereits bestehenden Multiplikatoren übernehmen.

- Im Vorfeld einer Netzwerkentwicklung sollte der politische Wille zur Bildung eines solchen Netzwerks deutlich werden und ein solches Netzwerk mit den notwendigen Ressourcen ausgestattet werden.

- Zur Bearbeitung spezifischer Problemfelder sollten innerhalb eines übergreifenden Netzwerks kleinere Arbeitsgruppen mit konkreten Zielen, z. B. settingbezogene Lösungen, arbeiten.

- Für eine effektive Netzwerkentwicklung und -steuerung ist angesichts der gegebenen Netzwerkkonfiguration die Schaffung einer administrativen Einheit zur Netzwerksteuerung, die unabhängig von den Netzwerkmitgliedern agiert und die keine kommerziellen Interessen verfolgt, empfehlenswert. Die koordinierende Organisation muss über ein hohes Maß an Kompetenzen der Netzwerksteuerung verfügen. Neben Maßnahmen der Vertrauensbildung gehören dazu die Kommunikation des Netzwerknutzens und des Ziels, die Koordination von Aufgaben, die Selektion von Netzwerkmitgliedern, die Allokation von Netzwerkressourcen, ein effektives Zeitmanagement (Vor- und Nachbereitung von Netzwerktreffen), die Lösung von Konflikten und Konkurrenzsituationen sowie die Berücksichtigung des in Netzwerken häufig auftretenden Trittbrettfahrerproblems.

- Zur Messung der Zielerreichung des Netzwerks hinsichtlich einer flächendeckenden und nachhaltigen Implementierung der Bewegungsempfehlungen sowie einer Qualitätsentwicklung ist eine wissenschaftliche Begleitung anzuraten.

\section{Fazit und Ausblick}

Die Untersuchung ermöglicht eine 1. differenzierte Betrachtung der Akteure bzw. Stakeholder der Bewegungsförderung in Deutschland und deren strukturellem Zusammenhang auf Basis von Expertenwissen. Bei der Einordnung der Ergebnisse ist zu berücksichtigen, dass im Rahmen der explorativen Vorgehensweise lediglich eine 1. Bestandsaufnahme vorgenommen wurde. Diese basiert auf subjektiven Meinungen und Wahrnehmungen von Experten, deren Auswahl zudem einem „Selection Bias“ unterliegen kann. Diese Einschränkungen bringen mit sich, dass die Ergebnisse keine vollständige und lückenlose Darstellung der bestehenden Situation darstellen. Allerdings erfüllen sie den Zweck der Untersuchung, der darin liegt, einen Überblick und Orientierung über die Landschaft der Bewegungsförderung in Deutschland und mögliche Ansätze einer Entwicklung und Gestaltung zu geben.

Die Ergebnisse offenbaren, dass die Bewegungsförderungslandschaft einerseits viele Gesellschaftsbereiche umfasst und andererseits durch eine große Heterogenität der Akteure geprägt ist. Die relevanten Akteure sind Organisationen, Personen,
Berufsgruppen und soziale Institutionen, die auf nationaler Ebene, Landes- und Regional-/Kommunalebene agieren. Die Mehrzahl der relevanten Akteure wird dabei im Bereich Politik und Verwaltung verortet. Aus dem Kreis der weit über 100 Akteure ließen sich verschiedene Schlüsselpositionen, Multiplikatoren und potenzielle Multiplikatoren identifizieren.

Die Analyse der strukturellen Vernetzung der identifizierten Akteure zeigte, dass die Kooperationsstrukturen verhältnismäßig schwach ausgeprägt sind. Trotzdem sind vereinzelte Konflikte und Konkurrenzsituationen zu erkennen, deren Adressierung eine Herausforderung im Rahmen einer Netzwerkentwicklung darstellt. Eine effektive Steuerung eines nationalen Netzwerks der Bewegungsförderung bedarf einer konkreten Zielsetzung, Ressourcenbereitstellung sowie eines zu schaffenden Koordinierungsakteurs, der über Kompetenzen der Netzwerkentwicklung und -steuerung verfügt bedarf. Dabei sind die im Rahmen der Untersuchung identifizierten Voraussetzungen, Nutzen und Barrieren der Netzwerkentwicklung zu berücksichtigen.

Die erste Aufgabe, die sich im Anschluss an diese Studie ergibt, ist die Entwicklung eines kooperativen und deutschlandweit agierenden Netzwerks der Bewegungsförderung (vgl. auch die Empfehlungen von [7]). Vorrangige Aufgabe wäre zunächst die Disseminierung der NEBB auf Grundlage eines Kommunikationskonzepts. In diesem Zusammenhang könnte zunächst ein Pilotprojekt der Disseminierung der NEBB auf regionaler Ebene oder am Beispiel noch nicht aktivierter Akteure durchgeführt werden. Im einem weiteren Schritt sollten auf den NEBB aufbauend nachhaltige Maßnahmen einer Bewegungsförderung entwickelt werden und in einer übergreifenden Strategie zusammengeführt werden, um die Akteure auf Landes- und kommunaler Ebene in ihrer Arbeit mit den Zielgruppen zu unterstützen.

\section{Interessenkonflikt}

Der Deutsche Verband für Gesundheitssport und Sporttherapie (DVGS) e. V. ist als Projektnehmer (Projektleitung: Frau Angelika Baldus) und Fachverband selbst einer der Akteure für Bewegungsförderung in Deutschland. Stefan Peters leitet das Wissenschaftsressort des DVGS e. V.

\section{Danksagung}

Das Projekt SAMBA wurde gefördert vom Bundesministerium für Gesundheit (BMG). Die finanzielle Förderung erfolgte aufgrund eines Beschlusses des Deutschen Bundestages durch die Bundesregierung.

\section{Autorinnen / Autoren}

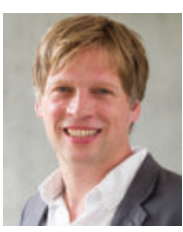

\section{Dr. Hagen Wäsche}

Institut für Sport und Sportwissenschaft Karlsruher Institut für Technologie Engler-Bunte-Ring 15 76131 Karlsruhe

E-Mail: hagen.waesche@kit.edu 


\section{Literatur}

[1] Borgatti SP, Everett MG, Johnson JC. Analyzing social networks. Los Angeles, CA: Sage; 2013

[2] Bornstein DB, Pate RR, Pratt M. A review of the national physical activity plans of six countries. Journal of Physical Activity and Health. 2009; 6 (2): $245-264$

[3] Flick U: Designing Qualitative Research. London: Sage; 2007

[4] Gamper M, Schönhuth M, Kronenwett M. Bringing Qualitative and Quantitative Data Together: Collecting Network Data with the Help of the Software Tool VennMaker. In: Safar M, Mahdi K (Hg). Social Networking and Community Behavior Modeling: Qualitative and Quantitative Measures. Hershey, PA: IGI Publi-shing; 2012: 193-213

[5] GKV Spitzenverband et al: Bundesrahmenempfehlungen der NPK nach $\S 20$ d Abs. 3 SGB V. Zugriff am 06.03.2017 unter https: / /www.gkv-spi tzenver-band.de/media/dokumente/presse/pressemitteilungen/2016/P raevention_NPK_BRE_verabschiedet_am_19022016.pdf

[6] Gläser J, Laudel G. Experteninterviews und qualitative Inhaltsanalyse. Wiesbaden: VS Verlag für Sozialwissenschaften; 2004

[7] Gohres H, Kolip P. Strukturen der Bewegungsförderung in Deutschland: Hand-lungsempfehlungen zur strukturellen Weiterentwicklung als Ergebnisse einer Del-phi-Befragung. Prävention und Gesundheitsförderung. 2017; 12 (3): 201-210

[8] Krug S et al. Körperliche Aktivität. Ergebnisse der Studie zur Gesundheit Erwachse-ner in Deutschland (DEGS1). Bundesgesundheitsblatt. 2013; 56: 765-771

[9] Mayring P. Qualitative Inhaltsanalyse. Grundlagen und Techniken. Weinheim: Beltz; 2003

[10] ParticipACTION Advisory Groups. Active Canada 20/20: A Physical Activity Strategy and Change Agenda for Canada - Creating a Culture of an Active Nation. 05 / 2012. Zugriff am 11.09.2015 unter http: / /www.acti vecanada2020.cal

[11] Peters S, Wäsche H. Projekt SAMBA: Systematische Erfassung relevanter Akteure, Berufsgruppen sowie künftiger Multiplikatoren in der
Bewegungsförderung zur Analyse und Entwicklung eines interdisziplinären Netzwerks zur nachhaltigen Bewegungsförderung. Bewegungstherapie \& Gesundheitssport. 2017; 33 (3): 114-118

[12] Physical Activity Guidelines Advisory Committee. Physical Activity Guidelines Advi-sory Committee Report, 2008. Washington, DC: U.S. Department of Health and Hu-man Services; 2008

[13] Provan KG, Kenis PN. Modes of network governance: Structure, management, and effectiveness. Journal of Public Administration Research and Theory. 2008; 18 (2): 229-252

[14] Reiner M, Niermann C, Jekauc D, Woll A. Long-term health benefits of physical ac-tivity-a systematic review of longitudinal studies. BMC Public Health. 2013; 13 (1): 813

[15] Rütten A, Pfeifer K (Hg). Nationale Empfehlungen für Bewegung und Bewegungs-förderung. Erlangen: FAU Erlangen-Nürnberg; 2016

[16] Rütten A, Abu-Omar K, Lampert T, Ziese T. Körperliche Aktivität. Berlin: RKI; 2005. Zugriff am 07.07.2018 unter: https: / /edoc.rki.de/bitstream/han

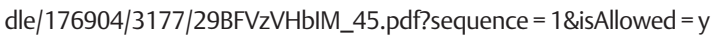

[17] Sallis JF, Owen N, Fisher EB. Ecological models of health behavior. In Glanz K, Rimer BK, Visnawath K (Eds). Health Behavior and Health Education (4th ed.). San Francisco: Jossey Bass; 2008: 456-485

[18] Spence JC, Faulkner G, Bradstreet CC et al. Active Cana-da 20/20: a physical activi-ty plan for Canada. Canadian Journal of Public Health. 2015; 106 (8): e470-e473

[19] Wäsche H, Woll A, Dickson G, Brandes U. Social network analysis in sport research: An emerging paradigm. European Journal for Sport and Society. 2017; 14: 138-165

[20] WHO: Global recommendations on physical activity for health. Genf: WHO; 2010. Zugriff am 31.10.2017 unter: http://apps.who.int/iris/bit stream/10665/44399/1/9789241599979_eng.pdf

[21] WHO: Physical activity strategy for the WHO European Region 2016 - 2025. Ko-penhagen: WHO; 2016. Zugriff am 07.07.2018 unter http: / /www.euro.who.int/__data/assets/pdf_file/0014/311360/Physi cal-activity-strategy-2016-2025.pdf?ua $=1$ 